\title{
Antiobesity, Regulation of Lipid Metabolism, and Attenuation of Liver Oxidative Stress Effects of Hydroxy- $\alpha$-sanshool Isolated from Zanthoxylum bungeanum on High-Fat Diet-Induced Hyperlipidemic Rats
}

\author{
Li Wang $\left(\mathbb{D}\right.$, Wenxiang Fan $\left(\mathbb{D}\right.$, Mengmeng Zhang $\left(\mathbb{D}\right.$, Qing Zhang $\mathbb{D}$, Lin $\operatorname{Li}^{(\mathbb{D}}$, \\ Jiaolong Wang $\mathbb{D}^{D}$, Lei Zhu $\mathbb{D}$, Daneng Wei, Wei Peng $\mathbb{D}$, and Chunjie Wu $\mathbb{D}$ \\ School of Pharmacy, Chengdu University of Traditional Chinese Medicine, Chengdu, China \\ Correspondence should be addressed to Wei Peng; pengwei@cdutcm.edu.cn and ChunjieWu; wucjcdtcm@163.com
}

Received 8 January 2019; Revised 24 May 2019; Accepted 8 July 2019; Published 27 August 2019

Guest Editor: Sergey Bolevich

Copyright ( 2019 Li Wang et al. This is an open access article distributed under the Creative Commons Attribution License, which permits unrestricted use, distribution, and reproduction in any medium, provided the original work is properly cited.

Zanthoxylum bungeanum is a traditional Chinese medicine (TCM) used to relieve pain, dispel dampness, stop diarrhea, and prevent itching. The aim of this study was to investigate the antiobesity and hypolipidemic effects of hydroxy- $\alpha$-sanshool (HAS) isolated from Z. bungeanum on hyperlipidemic rats. Wistar rats $(n=48)$ were randomly divided into six groups: (1) normal diet rats (ND), (2) high-fat diet- (HFD-) treated rats, (3) HFD+fenofibrate-treated rats (HFD+FNB), (4) HFD+low dose of HAStreated rats (HFD+LD, $9 \mathrm{mg} / \mathrm{kg}$ ), (5) HFD+middle dose of HAS-treated rats (HFD+MD, $18 \mathrm{mg} / \mathrm{kg}$ ), and (6) HFD+high dose of HAS-treated rats $(\mathrm{HFD}+\mathrm{HD}, 36 \mathrm{mg} / \mathrm{kg})$. The body weight and food intake of the rats were recorded during the treatment period. After 4 weeks of HAS treatment, abdominal adipose tissues were observed and total cholesterol (T-CHO), triglycerides (TG), high-density lipoprotein (HDL) cholesterol (HDL-C), and low-density lipoprotein (LDL) cholesterol (LDL-C) of serum and liver tissues were determined. Furthermore, histochemical examinations using oil red $\mathrm{O}$ and hematoxylin-eosin staining (H\&E) were carried out and levels of malondialdehyde (MDA) and glutathione (GSH) and activities of superoxide dismutase (SOD) in the liver were determined. After HFD feeding, the body weight gain and food efficiency ratio of HFD rats were significantly enhanced ( $p<0.05 \mathrm{vs}$. ND rats) and HAS treatment $(18$ and $36 \mathrm{mg} / \mathrm{kg})$ significantly decreased the body weight gain and food efficiency ratio ( $p<0.05 v s$. HFD rats). In addition, HAS treatment could decrease the abdominal adipose tissues and liver adipocytes. Furthermore, HAS treatment significantly decreased the T-CHO, TG, and LDL-C, whereas it increased HDL-C $(p<0.05 v$ s. HFD rats) in serum and the liver. HAS treatment increased the GSH level and SOD activity in the liver $(p<0.05 v s$. HFD rats), whereas it decreased the levels of MDA $(p<0.05 v s$. HFD rats). mRNA analyses suggested that HAS treatment increases the expression of Pparg (proliferator-activated receptor $\gamma$ ) and Apoe (peroxisome apolipoprotein E). Immunohistochemistry and Western blotting indicated that HAS stimulation increased the levels of PPAR $\gamma$ and APOE in the liver, as a stress response of the body defense system. These results revealed that HAS exerts antiobesity and hypolipidemic activities in HFD rats by reducing liver oxidative stress and thus could be considered as a potential candidate drug to cure or prevent obesity and hyperlipidemia.

\section{Introduction}

Cardiovascular diseases (CVDs), such as atherosclerosis (AS) and coronary heart disease (CHD), have become a global epidemic with high morbidity and mortality. Previous studies have shown that obesity and hyperlipidemia are positive risk factors for the initiation of atherosclerosis, which lead to a series of cardiovascular complications. Hyperlipidemia is a metabolic disorder disease that involves abnormally high levels of blood lipids and lipoproteins [1-3]. With the globalization of Western diet, a high-fat and high-cholesterol diet is believed to be one of the most important causes of the high incidence of obesity and hyperlipidemia worldwide [4]. Furthermore, the increased formation of free radicals and 


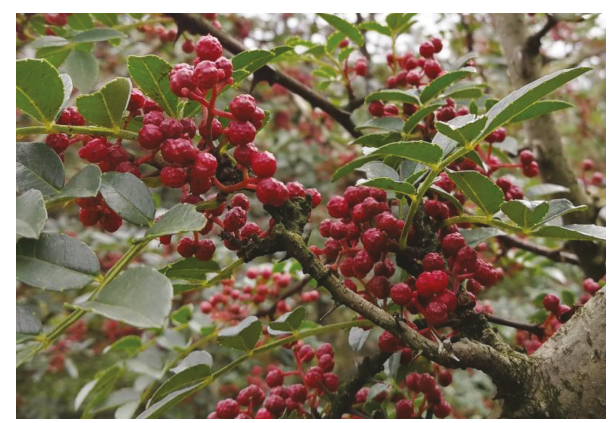

(a)

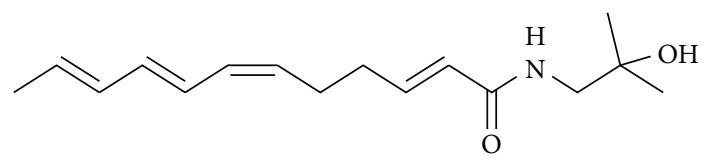

(b)

Figure 1: Zanthoxylum bungeanum (a) and hydroxy- $\alpha$-sanshool (b).

reactive oxygen species (ROS) participates in cardiac dysfunction, CVD progression, and cardiac apoptosis [3]. Oxidative stress occurs when the concentration of free radicals exceeds a critical level and the homeostasis of the body is disturbed [5]. Malondialdehyde (MDA), as an iconic product of oxidative stress, accumulates in the body and is toxic to cells, while superoxide dismutase (SOD) is an important part of the oxidation protection system that catalyzes the dismutation of the superoxide radical, thereby alleviating oxidative stress $[5,6]$. The tripeptide glutathione (GSH) is also the most important cellular antioxidant [4]. Thus, attenuation of oxidative stress may be an effective preventive measure against hyperlipidemia. Current antihyperlipidemic medicines mainly include statins, fibrates, and bile acid chelators; however, they are inefficient in regulating lipid metabolism and attenuating oxidative stress. In addition, they also cause serious adverse effects such as rhabdomyolysis [3]. Therefore, the identification of a lipid-lowering drug with antioxidant potential from plants has attracted research interest. A recent study indicated that capsaicin extracted from the red chili pepper is beneficial for hyperlipidemia and atherosclerosis in guinea pigs fed a high-fat diet [7]. Furthermore, tetrahydrocurcumin intervention therapy has been proved to have therapeutic effects against obesity and hepatic steatosis [8].

Zanthoxylum bungeanum Maxim ( $Z$. bungeanum) (Figure 1(a)), belonging to the family of Rutaceae, is used as a seasoning spice in China because of its unique aroma and taste $[9,10]$. Z. bungeanum is also used as an effective traditional Chinese medicine to relieve pain, dispel dampness, stop diarrhea, and prevent itching $[10,11]$. Previous phytochemical research reported that the main constituents of $Z$. bungeanum are unsaturated fatty acid amides of sanshool, such as hydroxy- $\alpha$-sanshool, hydroxy- $\beta$-sanshool, and hydroxy- $\gamma$-sanshool $[9,11]$. Pharmacological investigations revealed that $Z$. bungeanum possesses various pharmacological activities, in particular hypolipidemic and hypoglycemic effects $[11,12]$. However, no active monomers corresponding to the hypolipidemic effect of $Z$. bungeanum have been reported. Consequently, as part of our continuing investigation into $Z$. bungeanum, we aimed to investigate the antiobesity and hypolipidemic effects of hydroxy- $\alpha$-sanshool (HAS) (Figure 1(b)) isolated from Z. bungeanum on hyperlipidemic rats induced by high-fat diets. The present study would be beneficial for the development of HAS as a candidate drug to treat obesity and hyperlipidemia in the clinic.

\section{Materials and Methods}

2.1. Chemicals. The total cholesterol (T-CHO), triglycerides (TG), high-density lipoprotein (HDL) cholesterol (HDL-C), low-density lipoprotein (LDL) cholesterol (LDL-C), superoxide dismutase (SOD), malondialdehyde (MDA), tripeptide glutathione $(\mathrm{GSH})$, and coomassie bright blue (CBB) kits were purchased from Nanjing Jiancheng Bioengineering Institute (Nanjing, China); rabbit anti-PPAR $\gamma$ (proliferatoractivated receptor $\gamma$ ) and rabbit anti-APOE (peroxisome apolipoprotein E) antibodies were products of Bioss Antibodies (Beijing, China); GAPDH antibodies, radioimmunoprecipitation assay (RIPA) lysate buffer, bicinchoninic acid (BCA) protein quantitative kit, and SDS-polyacrylamide gel electrophoresis (SDS-PAGE) preparation kit were purchased from Multi Sciences (Hangzhou, China); RNA TRIzol Reagent was purchased from Servicebio Company (Wuhan, China); the RevertAid First Strand cDNA Synthesis Kit was purchased from Thermo Fisher (MO, USA); and the all other chemicals used in this study were of analytical reagent grade.

2.2. Plant Material. The dried fruits of $Z$. bungeanum were collected from Qingxi town, Hanyuan County (Ya'an, China), and identified by Professor Chunjie Wu (School of Pharmacy, Chengdu University of Traditional Chinese Medicine, Chengdu, China). The voucher specimen of the fruit of $Z$. bungeanum was deposited at our laboratory (no. 20180317-1\#).

2.3. Preparation of HAS. Fruits of $Z$. bungeanum were extracted twice with 20 volumes of $90 \%$ methanol $(v / v)$ using a $1.5 \mathrm{~h}$ reflux extraction and subsequently concentrated under reduced pressure at $45^{\circ} \mathrm{C}$. The concentrated extracts were then extracted by ethyl acetate three times and filtered, and then the solvent was vaporized to obtain the ethyl acetate fraction. The ethyl acetate fraction was then subjected to repeated column chromatography over silica gel (100-200 mesh) and eluted with petroleum ether-ethyl acetate $(3: 1$ $1: 2$ ) to yield four subfractions (sub-Fra. A-D). The sub-Fra. $\mathrm{C}$ was further subjected to preparative high-performance liquid chromatography (HPLC) with a CAPCELL PAK C18 MGII-F92539 $(250 \mathrm{~mm} \times 20 \mathrm{~mm}, 5 \mu \mathrm{m}$; OSAKA SODA Co. Ltd., Osaka, Japan) column and eluted with acetonitrile in water $(40: 60)$ to yield the HAS monomer. Furthermore, HPLC analysis with an HAS standard was carried out to determine the authenticity and purity of the HAS. The Shimadzu LC-2010A system (Shimadzu Co., Japan) with a CAPCELL PAK C18 MGII-S5 column $(4.6 \mathrm{~mm} \times 250 \mathrm{~mm}$, $5 \mu \mathrm{m}$; OSAKA SODA Co. Ltd.) was used to perform the HPLC analysis, and acetonitrile (A)/water $(\mathrm{B})=40: 60$ was used as the mobile phase. The separation temperature was 


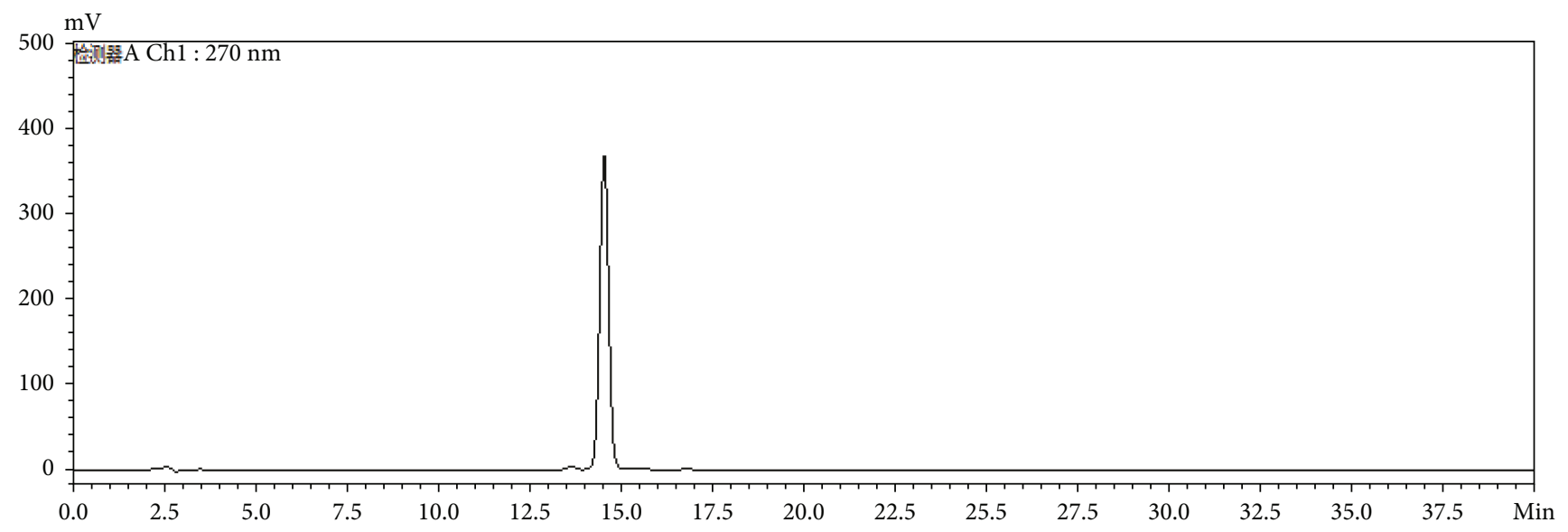

(a)

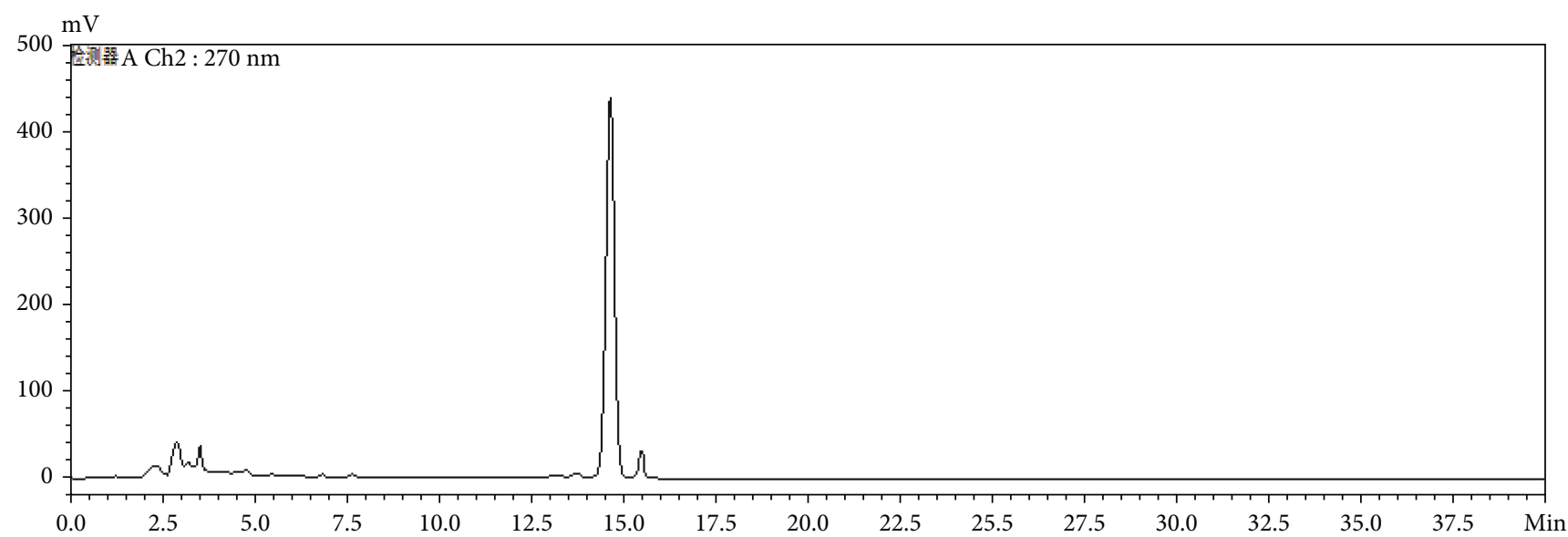

(b)

FIGURE 2: High-performance liquid chromatography (HPLC) analysis of the hydroxy- $\alpha$-sanshool (HAS) extracted from the fruit of $Z$. bungeanum. (a) HPLC chromatogram of the standard; (b) HPLC chromatogram of the HAS extracted from the fruit of Z. bungeanum.

maintained at a constant $25^{\circ} \mathrm{C}$ for $40 \mathrm{~min}$, with a flow rate of $1.0 \mathrm{~mL} / \mathrm{min}$ and an injection volume of $10 \mu \mathrm{L}$. Peaks were identified by comparing retention times and UV spectra with those of commercial standards and were quantified based on peak areas at $270 \mathrm{~nm}$; the purity of the HAS was over $96.0 \%$ (Figure 2).

2.4. Toxicity Tests. A total of 60 Wistar rats were randomly divided into six groups $(n=10)$. Rats in groups $1-5$ were orally administered $4.5,9,18,36$, and $72 \mathrm{mg} / \mathrm{kg}$ of HAS, respectively, and rats in the $6^{\text {th }}$ group received $20 \mathrm{~mL} / \mathrm{kg}$ of normal saline. The mortality rates of the testing rats within a $72 \mathrm{~h}$ observation period were recorded. Furthermore, our results indicated that neither death nor any abnormal neurobehaviors could be observed with the doses ranging from 4.5 to $72 \mathrm{mg} / \mathrm{kg}$ during $72 \mathrm{~h}$. Therefore, the $50 \%$ lethal dose $\left(\mathrm{LD}_{50}\right)$ was not obtained and the safe doses $(9,18$, and $36 \mathrm{mg} / \mathrm{kg}$ ) were selected as administration dosages in our present study for the testing rats.

2.5. Animal Protocols. All animal experiments were conducted under protocols approved by the experimental animal ethics committee of Chengdu University of Traditional
Chinese Medicine. Wistar rats $(220 \pm 20 \mathrm{~g})$ were purchased from the Chengdu Dashuo Experimental Animals Co. Ltd. (Chengdu, China) and housed in a facility maintained at a constant temperature $\left(22 \pm 1^{\circ} \mathrm{C}\right)$ and humidity $(55 \pm 10 \%)$ with an alternating 12 hour light/dark cycle. All rats were provided with ad libitum access to water and standard rodent chow for 1 week before performing the experiments. In total, 48 Wistar rats were randomly divided into six groups $(n=8)$. Besides the normal diet (ND) group, rats in the other five groups were fed with high-fat diet (HFD) feeds (consisting of basic feed (73\%), cholesterol (1.5\%), pig fat (10\%), egg yolk powder $(5 \%)$, sucrose $(10 \%)$, and bile salt $(0.5 \%))$.

After two weeks of HFD feeding, to evaluate whether the hyperlipidemic rats were prepared successfully, the total cholesterol (T-CHO), triglycerides (TG), high-density lipoprotein (HDL) cholesterol (HDL-C), and low-density lipoprotein (LDL) cholesterol (LDL-C) in the rats' serum were determined. Briefly, blood samples were collected from rat tail veins and centrifuged at $4^{\circ} \mathrm{C}(2500 \mathrm{rpm}, 15 \mathrm{~min})$ to provide the serum samples for the biochemical assays, which were performed using commercial testing kits according to the manufacturer's instructions. As shown in Figure 3, the levels of T-CHO $(p<0.05)$, TG $(p<0.05)$, and LDL-C 


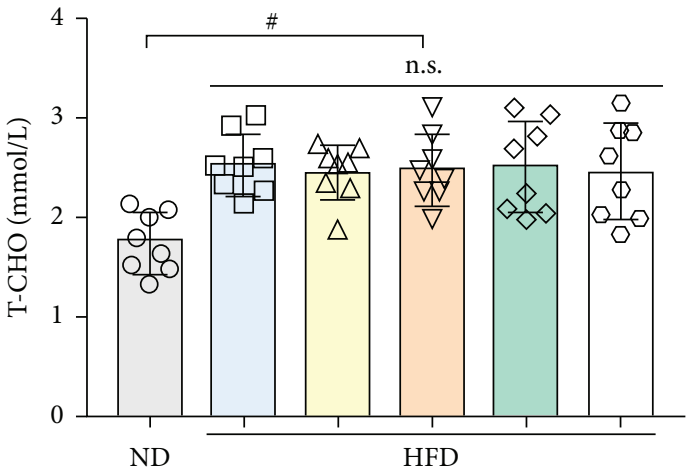

(a)

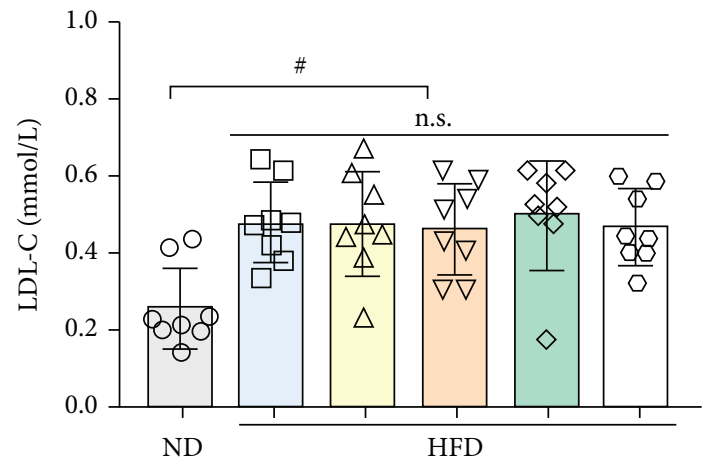

(c)

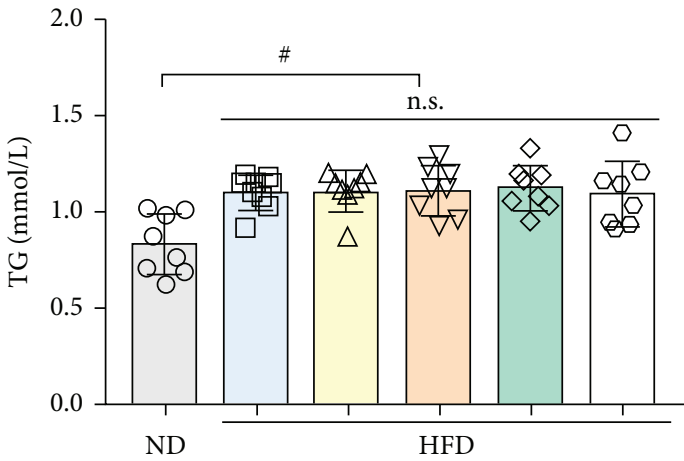

(b)

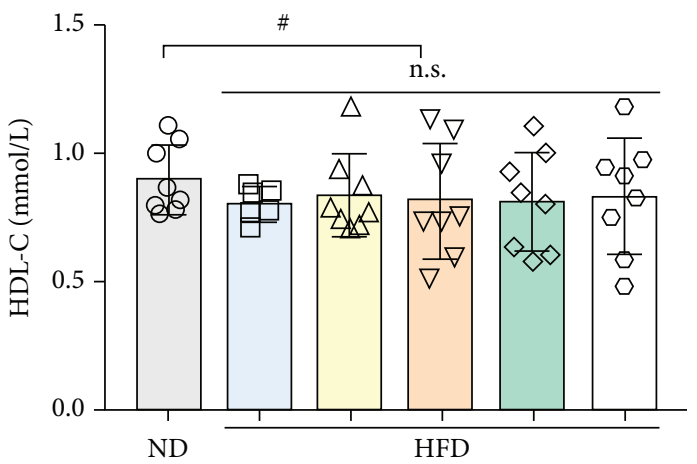

(d)

FIgure 3: Total cholesterol (T-CHO) (a), total glycerides (TG) (b), low-density lipoprotein cholesterol (LDL-C) (c), and high-density lipoprotein chol esterol (HDL-C) (d) in rat serum consuming a normal diet (ND) and a high-fat diet (HFD) for two weeks. Values are expressed as the mean \pm SD $(n=8),{ }^{\#} p<0.05 v s$. ND; n.s.: nonsignificant.

$(p<0.05)$ in serum increased significantly, whereas the HDL-C levels decreased significantly $(p<0.05)$, compared with those in the ND rats. These results suggested that the hyperlipidemic rats were prepared successfully and could be used for further experiments.

The HFD-treated rats were adjusted and subdivided into five groups ( $n=8$ in each group) according to the body weight of the rats and the biochemical values in serum. Subsequently, the HAS and fenofibrate (FNB) were dissolved in $0.5 \%$ carboxymethyl cellulose (CMC) - Na solution and all the tested drugs were administered orally; rats were treated with FNB (HFD+FNB, $18 \mathrm{mg} / \mathrm{kg}$ ), a low dose of HAS (HFD+ $\mathrm{LD}, 9 \mathrm{mg} / \mathrm{kg}$ ), a middle dose of HAS (HFD+MD, $18 \mathrm{mg} / \mathrm{kg}$ ), and a high dose of HAS (HFD+HD, $36 \mathrm{mg} / \mathrm{kg}$ ) for 4 weeks. Rats in the ND and HFD groups were treated with an equivalent volume of $0.5 \%$ CMC-Na solution. The rats' body weights were measured every week, and food intake was recorded every day during the treatment period. After four weeks of treatment, the rats were anesthetized with $2 \%$ $(v / v)$ sodium pentobarbital $(3 \mathrm{~mL} / \mathrm{kg})$ and then blood samples were collected from the abdominal aorta. The rats' abdomens were opened for observation and to isolate the total abdominal fat; all rats were then sacrificed by cervical dislocation after blood sampling. The liver tissues were collected rapidly and cut into three pieces; one piece was sectioned into small pieces $(1 \mathrm{~cm} \times 1 \mathrm{~cm})$ and fixed with $4 \%$ paraformaldehyde, one piece was frozen in liquid nitrogen, and the last piece was stored at $-20^{\circ} \mathrm{C}$ for further biochemical analyses.
In addition, blood samples were centrifuged at $2500 \mathrm{rpm}$ under $4^{\circ} \mathrm{C}$ for $15 \mathrm{~min}$ to acquire serum samples and subsequently stored at $-70^{\circ} \mathrm{C}$ for subsequent biochemical analyses. The experimental scheme is shown in Figure 4.

2.6. Biochemical Analysis in Serum and the Liver. The contents of HDL-C and LDL-C in serum and T-CHO and TG in the serum and liver tissues were measured using commercially available kits according to the manufacturer's instructions (Nanjing Jiancheng Bioengineering Institute), and a microplate reader was used to read the optical density (OD) values (BIO-BRI Technology Co. Ltd., Chengdu, China).

2.7. Oxidative Stress Marker Analysis in Liver Tissues. The levels of MDA and GSH and the activities of (SOD) in hepatic tissues were determined using commercially available colorimetric kits (Nanjing Jiancheng Bioengineering Institute) according to the manufacturer's instructions. Meanwhile, a CBB kit (Nanjing Jiancheng Bioengineering Institute) was used out to measure the total content of protein in $10 \%$ liver homogenate.

2.8. Oil Red O Staining. The liver tissue sections were fixed with formaldehyde for $10 \mathrm{~min}$, washed with distilled water, and soaked with $60 \%$ isopropyl alcohol. The fixed sections were then stained with oil red O solution for $10 \mathrm{~min}$ and subjected to hematoxylin staining. The lipid droplets were 


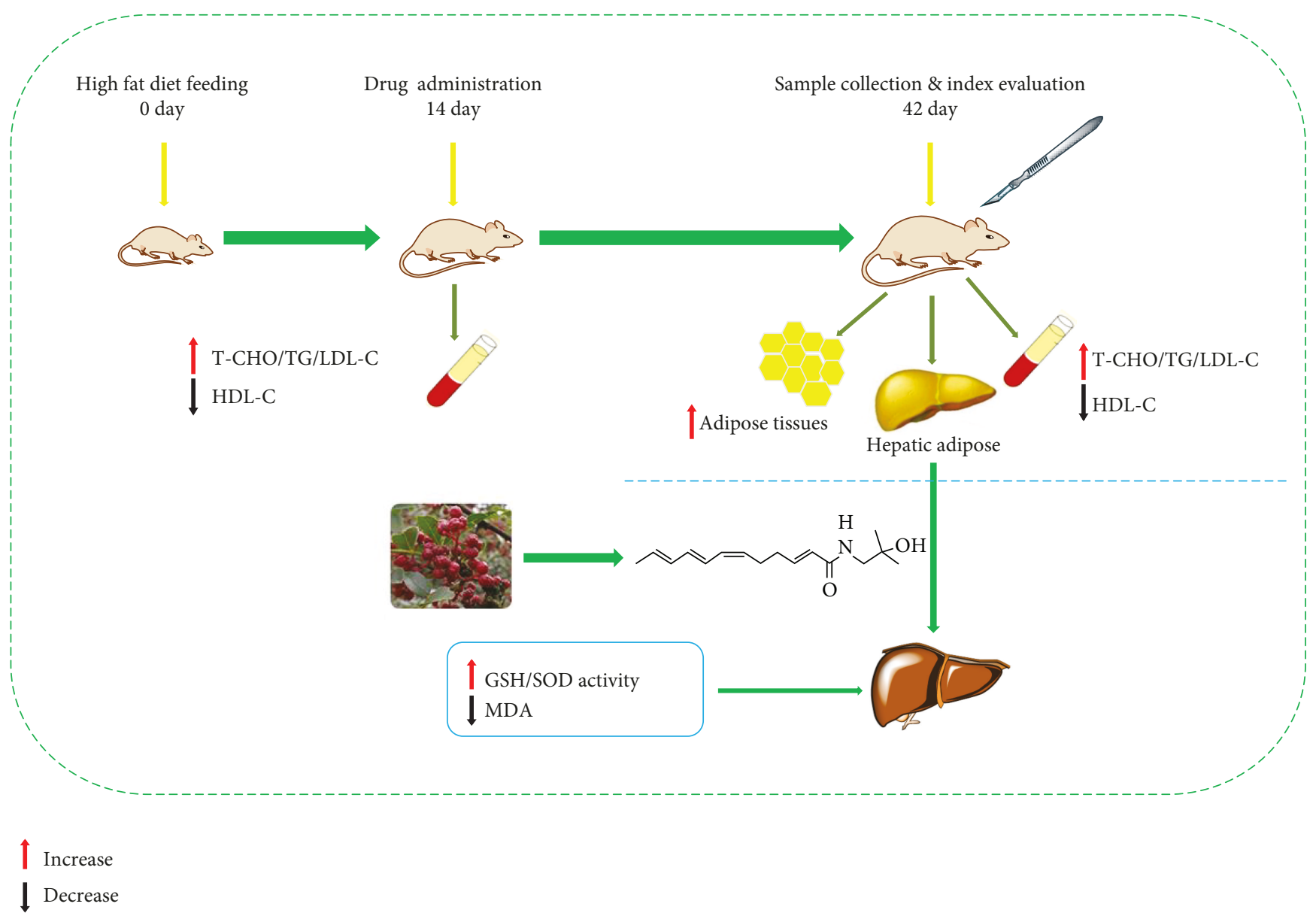

FIgURE 4: Schematic diagram of the experiment.

visualized using a digital trinocular camera microscope (BA400 Digital, McAudi).

2.9. Hematoxylin and Eosin Staining. The liver tissue sections were dewaxed, stained with hematoxylin for 10-20 min, and rinsed with tap water for 1-3 min. The sections were then incubated with hydrochloric acid alcohol for 5-10 s, rinsed with tap water, and placed in a weakly alkaline aqueous solution until a blue color appeared. The sections were then placed in $85 \%$ alcohol for $3-5 \mathrm{~min}$, stained with eosin for 3-5 min, and then washed for 3-5 s. Serial dilutions of ethyl alcohol were used for dehydration, and the sections were cleared in xylene and sealed using neutral glycerin. Images were acquired using a digital trinocular camera microscope (BA400 Digital, McAudi).

2.10. Immunohistochemistry (IHC). The liver tissue sections were dewaxed and incubated in 3\% methanol-hydrogen peroxide $(3: 97, v / v)$ for $10 \mathrm{~min}$ and then washed with phosphate-buffered saline (PBS) three times for $5 \mathrm{~min}$ each time. The sections were then immersed in citrate buffer ( $\mathrm{pH}$ 6.0), microwaved until boiling, repeated once, and washed with PBS two times for 5 min each time. After blocking with goat serum at room temperature for $20 \mathrm{~min}$, the tissue sections were incubated with rabbit anti-PPAR $\gamma$ (proliferator-activated receptor $\gamma)(1: 50$; cat. no. bs-0167R,
Bioss) and rabbit anti-APOE (peroxisome apolipoprotein E) antibodies (1:100; cat. no. bs-3614R, Bioss) at $4^{\circ} \mathrm{C}$ overnight, followed by incubation with a secondary antibody (cat. no. SP-9001, Beijing Zhongshan Jinqiao Biological Co. Ltd., Beijing, China) at $37^{\circ} \mathrm{C}$ for $30 \mathrm{~min}$. Specific labeling was visualized using a $3,3^{\prime}$-diaminobenzidine (DAB) kit and appeared yellow or brownish yellow, while cell nuclei were counterstained with hematoxylin (blue staining). Images were captured under a digital trinocular camera microscope (BA400 Digital, McAudi), and in each slide, three random images of the liver were obtained to determine the intensity of staining. IHC staining was expressed as the relative positive expression, and the mean density determined by the Image J software (version: 1.51, National Institutes of Health, $\mathrm{MD}$, USA) was used to compare the potential differences between groups.

2.11. Quantitative Real-Time PCR. The levels of Apoe mRNA and Pparg mRNA expression in the liver were measured by the quantitative real-time PCR. The liver tissue was homogenized, and total RNA was isolated using the TRIzol Reagent (G3013, Servicebio) according to the manufacturer's instructions. To synthesize cDNA, $2 \mu \mathrm{g}$ of total RNA was mixed with $1 \mu \mathrm{L}$ of oligo (dT) 18 and diethyl pyrocarbonatetreated water to a final volume of $12 \mu \mathrm{L}$. The mixture was incubated at $65^{\circ} \mathrm{C}$ for $5 \mathrm{~min}$ and cooled on the ice rapidly. 
TABLE 1: Sequences of primers used in qRT-PCR.

\begin{tabular}{|c|c|c|c|}
\hline Genes & & GenBank number & Fragment length (bp) \\
\hline \multicolumn{4}{|l|}{ Gapdh } \\
\hline F: & CTGGAGAAACCTGCCAAGTATG & \multirow{2}{*}{ NM_017008.4 } & \multirow{2}{*}{138} \\
\hline $\mathrm{R}:$ & GGTGGAAGAATGGGAGTTGCT & & \\
\hline \multicolumn{4}{|l|}{ Apoe } \\
\hline F: & TGACGGTACTGATGGAGGACACT & \multirow{2}{*}{ NM_001270681.1 } & \multirow{2}{*}{201} \\
\hline $\mathrm{R}:$ & CCAGCATGGTGTTTACCTCGTT & & \\
\hline \multicolumn{4}{|c|}{ 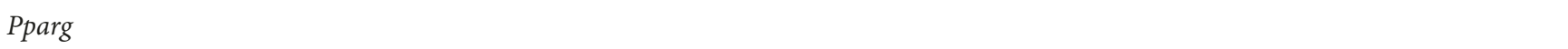 } \\
\hline F: & CCCTTTACCACGGTTGATTTC & \multirow{2}{*}{ NM_001145366.1 } & \multirow{2}{*}{321} \\
\hline $\mathrm{R}:$ & CTTCAATCGGATGGTTCTTCG & & \\
\hline
\end{tabular}

F: forward; R: reverse.

Then $4 \mu \mathrm{L}$ of $5 \mathrm{x}$ Reaction Buffer, $2 \mu \mathrm{L}$ of $10 \mathrm{mM}$ dNTP Mix, $1 \mu \mathrm{L}$ of RiboLock RNAase Inhibitor $(20 \mathrm{U} / \mu \mathrm{L})$, and $1 \mu \mathrm{L}$ of RevertAid M-MuLV Reverse Transcriptase $(200 \mathrm{U} / \mu \mathrm{L})$ were added successively. The mixture was incubated at $42^{\circ} \mathrm{C}$ for $60 \mathrm{~min}$, and the reaction was stopped by heat inactivation at $70^{\circ} \mathrm{C}$ for $5 \mathrm{~min}$. Quantitative real-time PCR was performed using a fluorescent quantitative PCR (Thermo Fisher Scientific). Reactions were carried out according to the following protocol: heating to $95^{\circ} \mathrm{C}$ for $10 \mathrm{~min}$, followed by 40 cycles of $95^{\circ} \mathrm{C}$ for $15 \mathrm{~s}$ and $60^{\circ} \mathrm{C}$ for $60 \mathrm{~s}$. Gapdh (Thermo Fisher) was used as the internal control gene to normalize the fold change in gene expression in the different groups. Primers for the target genes were designed by Sangon Bioengineering (Shanghai) Co. Ltd. (Shanghai, China) using Primer 3 software based on sequences retrieved from the GenBank database (Table 1).

2.12. Western Blot Analysis. Western blotting was used to measure the liver level of PPAR $\gamma$ and APOE. About $5 \mathrm{mg}$ of liver tissue was placed in radioimmunoprecipitation assay (RIPA) strong lysate buffer (RIPA+1\% PMSF) and pulverized. The total protein was extracted using a BCA protein quantitative kit (cat. no. 70-pq0011, Multi Science) according to the manufacturer's protocol. Protein samples were separated using $10 \%$ SDS-PAGE and then transferred to polyvinylidene fluoride (PVDF) membranes. Then the PVDF membranes were blocked by $5 \%$ skimmed milk. After that, the PVDF membranes were incubated by the primary antibodies of PPAR $\gamma$ (dilution: $1: 1000$ ), APOE (dilution: $1: 1000$ ), and GAPDH (dilution: $1: 1000$ ) overnight at $4^{\circ} \mathrm{C}$ and subsequently incubated by horse radish peroxidaseconjugated secondary antibody for $1 \mathrm{~h}$. The target protein bands were visualized by chemiluminescence detection with BeyoECL Star kits (Beyotime Biotech. Co., Haimen, China), and the protein band densities were determined by the ImageJ software (version: 1.51, National Institutes of Health); additionally, the protein expression levels of PPAR $\gamma$ and APOE were normalized to those of GAPDH.

2.13. Statistical Analysis. All results were expressed as the mean \pm the standard deviation (SD). The differences among the experimental groups were evaluated using one-way analysis of variance (ANOVA), and significant differences among the means were determined using Duncan's multiple- range tests. $p$ values less than 0.05 were considered statistically significant.

\section{Results}

3.1. Effects of HAS on Body Weight, Food Intake, Weight Gain, and the Food Efficiency Ratio. After 2 weeks of HFD feeding, the body weights of the HFD rats were significantly higher than those of the ND rats $(p<0.05)$ (Figure $5(\mathrm{a})$ ) and were similar to the positive drug-treated rats (fenofibrate, $18 \mathrm{mg} / \mathrm{kg}$ ). HAS could decrease the body weight of HFD rats at doses of 18 and $36 \mathrm{mg} / \mathrm{kg}(p<0.05)$ (Figure 5(a)). Furthermore, the results showed that the body weight gain (BWG) and food efficiency ratio (FER) were sharply increased by HFD feeding at the end of the experimental period (both $p<0.05$ ), compared with those of ND rats (Figures 5(b) and $5(\mathrm{~d}))$. Interestingly, both FNB $(18 \mathrm{mg} / \mathrm{kg})$ and HAS (18 and $36 \mathrm{mg} / \mathrm{kg}$ ) significantly reduced the BWG (Figure 5(b)) and FER (Figure 5(d)) of HFD-fed rats $(p<0.05)$. The food intake of the HFD rats was lower than that of the ND rats; however, HAS (18 and $36 \mathrm{mg} / \mathrm{kg}$ ) treatment had no obvious effect on the food intake of the HFD rats (Figure 5(d)).

3.2. Effect of HAS on Abdominal Adipose Tissue. As can be seen from Figure 6 and Table 2, after HFD feeding for 6 weeks, the HFD rats had much more white abdominal adipose tissue than the ND rats (Figures 6(a) and 6(b); $p<0.05)$. For the FNB-treated rats $(18 \mathrm{mg} / \mathrm{kg})$, the amount of white abdominal adipose tissue was reduced compared with that in the HFD rats $(p<0.05)$. Similar to FNB (Figure 6(c)), HAS at doses of 9, 18, and $36 \mathrm{mg} / \mathrm{kg}$ decreased the amount of white abdominal adipose tissues of HFD rats (Figures 6(d)-6(f); all $p<0.05)$.

3.3. Effect of HAS on Lipid Levels in Serum and Liver Tissues. From the results shown in Table 3, we observed that the levels of T-CHO and TG in the serum and liver tissues increased significantly (all $p<0.05$ ) compared with those in the ND rats. Importantly, the LDL-C level in serum increased $(p<0.05)$ sharply after HFD feeding, whereas the HDL-C levels markedly decreased $(p<0.05)$. Interestingly, treatment with FNB $(18 \mathrm{mg} / \mathrm{kg}, p<0.05)$ or HAS $(18$ and $36 \mathrm{mg} / \mathrm{kg}$; both $p<0.05)$ could significantly decrease the $\mathrm{T}-\mathrm{CHO}$ and TG levels in both serum and the liver, compared with those 


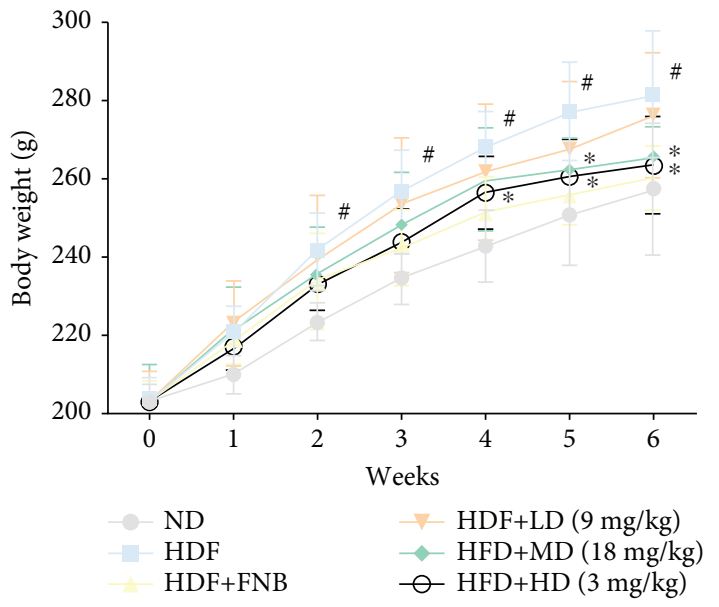

(a)

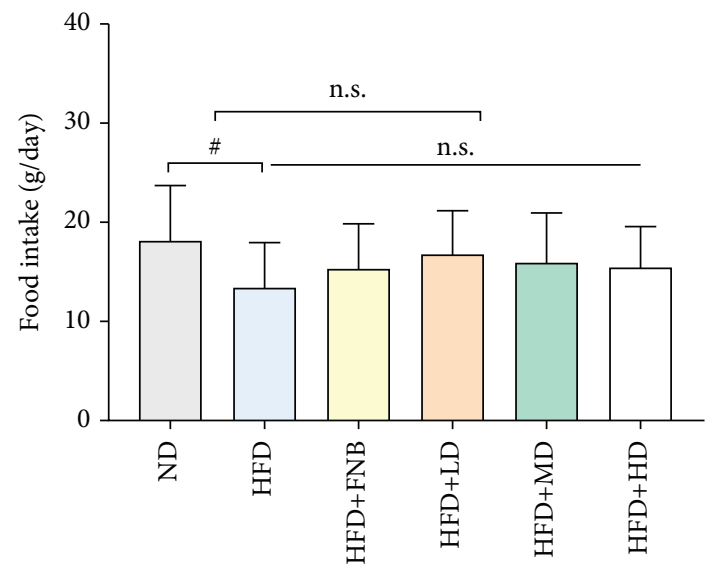

(c)

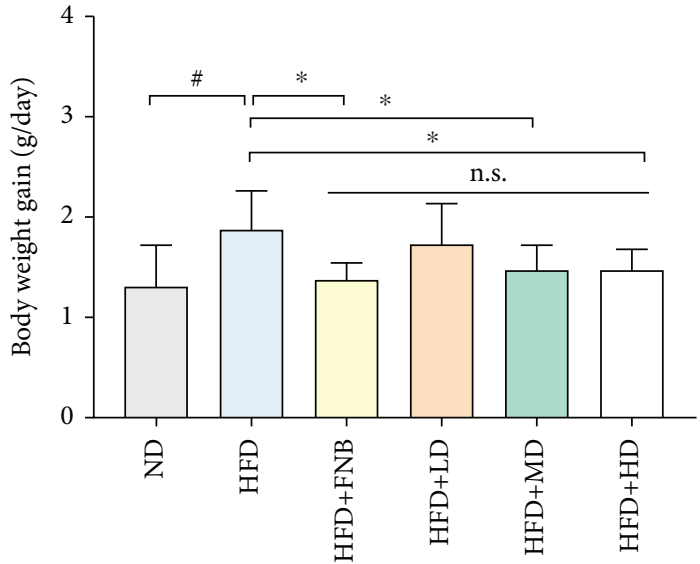

(b)

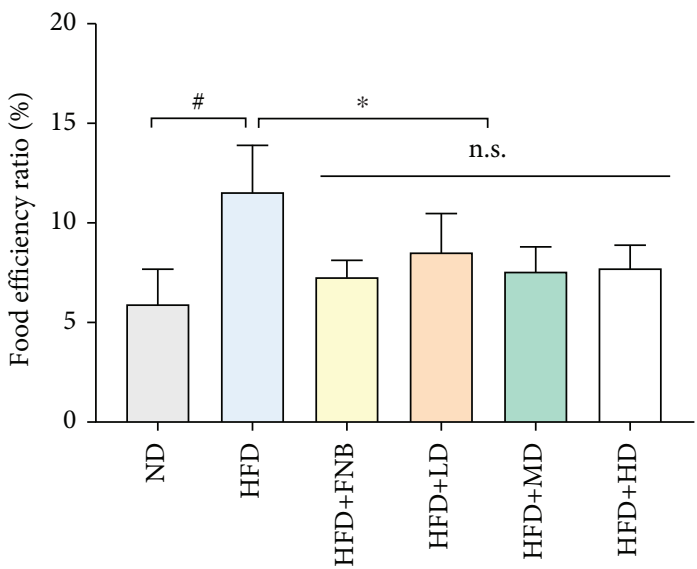

(d)

Figure 5: Effect of hydroxy- $\alpha$-sanshool (HAS) on body weight (a), body weight gain (b), food intake (c), and food efficiency ratio (d) in rats consuming a high-fat diet. We measured body weight every week for 6 weeks. The food efficiency ratio is the daily weight gain divided by the daily food intake. Values are expressed as the mean \pm SD $(n=8)$; ND: normal diet-treated rats; HFD: high-fat diet-treated rats; HFD+FNB: HFD supplemented with fenofibrate-treated rats; HFD+LD: rats treated with HFD supplemented with a low dose of HAS; HFD+MD: rats treated with HFD supplemented with a middle dose of HAS; HFD+HD: rats treated with HFD supplemented with a high dose of HAS; ${ }^{\#} p<0.05 v$ s. ND; ${ }^{*} p<0.05 v$ s. HFD; n.s.: nonsignificant.

in the rats in the HFD group. Furthermore, the results also indicated that FNB $(18 \mathrm{mg} / \mathrm{kg}, p<0.05)$ and HAS $(9,18$, and $36 \mathrm{mg} / \mathrm{kg}$; all $p<0.05)$ could ameliorate the increased LDL-C in the serum of HDF-fed rats, whereas FNB $(18 \mathrm{mg} / \mathrm{kg}, p<0.05)$ and HAS $(18 \mathrm{mg} / \mathrm{kg}, p<0.05)$ increased the serum level of HDL-C.

3.4. Results of the Histopathological Examinations. To determine the improving effects of HAS on the histopathological changes of liver tissues of hyperlipidaemic rats induced by HFD feeding, oil red $\mathrm{O}$ and $\mathrm{H} \& \mathrm{E}$ staining experiments were carried out. As shown in Figure 7, preadipocytes were stained with the fat-specific oil red $\mathrm{O}$ several days after the induction of hepatic adipocyte differentiation. The results revealed that HFD feeding markedly increase adipocyte differentiation compared with that in the ND rats (Figures $7(a)$ and $7(\mathrm{~b}))$. In addition, the results indicated that FNB $(18 \mathrm{mg} / \mathrm{kg}$, Figure $7(\mathrm{c}))$ and HAS $(9,18$, and $36 \mathrm{mg} / \mathrm{kg}$, Figures $7(\mathrm{~d})-$ $7(f))$ significantly decreased the adipocyte ratio in the liver tissues of HFD rats.

The results of the histopathological examinations of liver tissues using H\&E staining are shown in Figure 8. In the ND group, normal liver architecture and hepatic lobules were observed (Figure $8(a)$ ), while liver necrosis, interstitial edema, vacuolization of hepatocytes, and severe accumulation of lipid droplet in hepatocytes were observed in the HFD-fed rats (Figure 8(b)). However, FNB treatment $(18 \mathrm{mg} / \mathrm{kg})$ alleviated the pathological changes in liver tissues; especially, no obvious lipid droplets in hepatocyte were observed (Figure 8(c)). Similar to the effects of FNB, HAS $(9,18$, and $36 \mathrm{mg} / \mathrm{kg})$ also ameliorated lipid droplet accumulation in hepatocytes of HFD-fed rats (Figures 8(d)-8(f)).

3.5. Effect of HAS on Oxidative Stress Markers in Liver Tissues. The effects of HAS on MDA, GSH, and SOD in liver tissues are shown in Table 4. The results showed that HFD 


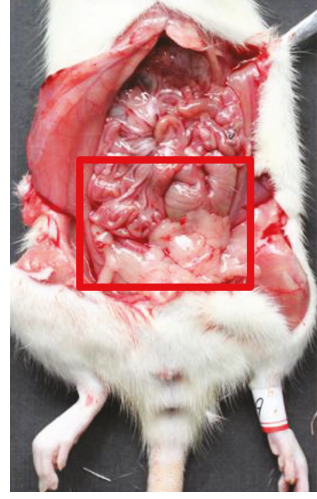

(a)

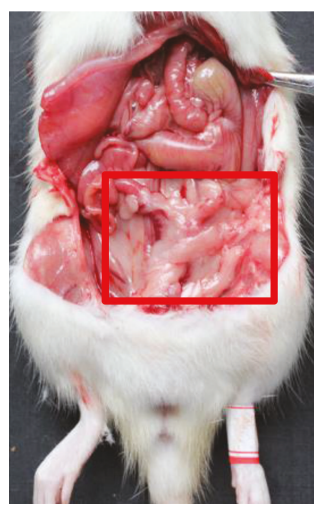

(d)

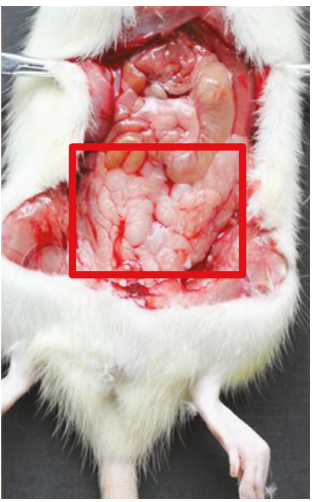

(b)

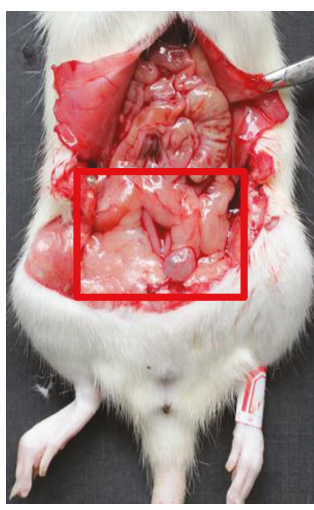

(e)

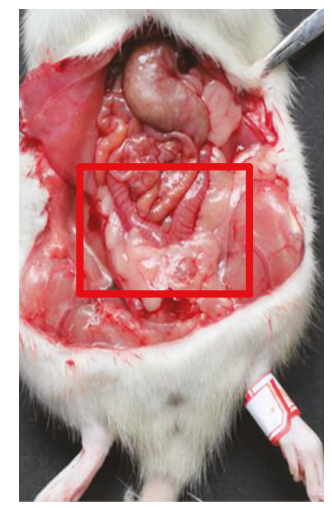

(c)

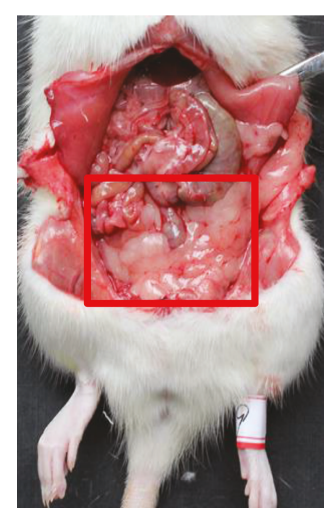

(f)

FIGURE 6: Effect of HAS on the abdominal adipose tissue in HFD rats. (a-f) represent the amount of abdominal adipose tissues of rats in the ND, HFD, HFD+FNB, HFD+LD, HFD+MD, and HFD+HD groups, respectively. ND: normal diet-treated rats; HFD: high-fat diet-treated rats; HFD+FNB: HFD supplemented with fenofibrate-treated rats; HFD+LD: rats treated with HFD supplemented with a low dose of HAS; HFD+MD: rats treated with HFD supplemented with a middle dose of HAS; HFD+HD: rats treated with HFD supplemented with a high dose of HAS.

TABLE 2: Effect of HAS on abdominal adipose tissue.

\begin{tabular}{lcc}
\hline Groups & $\begin{array}{c}\text { Abdominal adipose } \\
\text { tissue }(\mathrm{g})\end{array}$ & $\begin{array}{c}\text { Abdominal adipose tissue/final } \\
\text { body weight }(\%)\end{array}$ \\
\hline ND & $6.06 \pm 0.80$ & $2.35 \pm 0.28$ \\
HFD & $9.53 \pm 0.90^{\#}$ & $3.39 \pm 0.31^{\#}$ \\
HFD+FNB & $6.69 \pm 0.58^{*}$ & $2.57 \pm 0.20^{*}$ \\
HFD+LD & $7.96 \pm 0.71^{*}$ & $2.88 \pm 0.22^{*}$ \\
HFD+MD & $6.84 \pm 0.53^{*}$ & $2.58 \pm 0.26^{*}$ \\
HFD+HD & $6.55 \pm 0.39^{*}$ & $2.49 \pm 0.21^{*}$ \\
\hline
\end{tabular}

Values are expressed as mean $\pm \mathrm{SD}(n=8) ;{ }^{\#} p<0.05 v s$. the ND group and ${ }^{*} p<0.05 v s$. the HFD group. HAS: hydroxy- $\alpha$-sanshool; ND: normal diet; HFD: high-fat diet; FNB: fenofibrate; LD: low dose of HAS; MD: medium dose of HAS; HD: high dose of HAS.

feeding decreased the levels of SOD and GSH and increased that of MDA in liver tissues (all $p<0.05$ ), compared with those in the ND rats. Interestingly, the results suggested that the HAS and FNB could increase the levels of GSH and SOD and decrease the levels of MDA (all $p<0.05$ ).

3.6. Effect of HAS on the Levels of PPAR $\gamma$ and APOE in Liver Tissues through IHC. As shown in Figure 9(a), the PPAR $\gamma$ and APOE protein levels were enriched in the nuclear frac- tion and in the cytoplasmic fraction in liver cells using different doses of HFD+FNB and HFD+HAS $(9 \mathrm{mg} / \mathrm{kg}, 18 \mathrm{mg} / \mathrm{kg}$, and $36 \mathrm{mg} / \mathrm{kg}$ ) compared with those in the HFD groups (Figure 9(b), all $p<0.05$ ). The increased levels of PPAR $\gamma$ suggested that the HAS might activate the PPAR $\gamma$ pathway. Furthermore, the results also indicated that HAS and FNB could increase the expression levels of APOE compared with those in the HFD rats (Figure 9(b)).

3.7. Effect of HAS on the Transcription of Genes Involved in Lipid Metabolism in Liver Tissues. The mRNA expression of adipocyte markers, such as Apoe, was decreased in the liver tissues of HFD rats compared with those in the ND rats $(p<0.05$, Figure 10(a)), while the changes in Pparg expression were nonsignificant (Figures 10(a) and 10(b)). Furthermore, the results indicated that $\operatorname{HAS}(9,18$, and $36 \mathrm{mg} / \mathrm{kg}$; all $p<0.05$ ) could increase the expression of Pparg and Apoe mRNA compared with that in the HFD rats. FNB could also increase the expression of the Apoe mRNA compared with that in the HFD rats (Figure 10(b), $p<0.05$ ), while the change in Pparg expression was nonsignificant (Figure 10(a)).

3.8. Effect of HAS on PPAR $\gamma$ and APOE Levels in Liver Tissue. As shown in Figure 10, the levels of PPAR $\gamma$ in the HFD group were significantly lower than those in the ND group under 
TABLE 3: Effect of HAS on lipid levels in serum and liver tissues of HFD rats.

\begin{tabular}{|c|c|c|c|c|c|c|}
\hline \multirow[b]{2}{*}{ Groups } & \multicolumn{4}{|c|}{ Serum } & \multicolumn{2}{|c|}{ Liver } \\
\hline & $\begin{array}{l}\text { Total cholesterol } \\
(\mathrm{mmol} / \mathrm{L})\end{array}$ & $\begin{array}{l}\text { Triglyceride } \\
(\mathrm{mmol} / \mathrm{L})\end{array}$ & $\begin{array}{l}\text { LDL cholesterol } \\
(\mathrm{mmol} / \mathrm{L})\end{array}$ & $\begin{array}{l}\text { HDL cholesterol } \\
(\mathrm{mmol} / \mathrm{L})\end{array}$ & $\begin{array}{l}\text { Total cholesterol } \\
\text { (mmol/gprot) }\end{array}$ & $\begin{array}{c}\text { Triglyceride } \\
\text { (mmol/gprot) }\end{array}$ \\
\hline ND & $1.76 \pm 0.28$ & $0.88 \pm 0.07$ & $0.25 \pm 0.07$ & $1.10 \pm 0.16$ & $0.15 \pm 0.01$ & $0.22 \pm 0.02$ \\
\hline HFD & $2.80 \pm 0.52^{\#}$ & $1.84 \pm 0.18^{\#}$ & $0.98 \pm 0.16^{\#}$ & $0.93 \pm 0.11^{\#}$ & $0.69 \pm 0.16^{\#}$ & $0.43 \pm 0.07^{\#}$ \\
\hline $\begin{array}{l}\text { HFD } \\
+ \text { FNB }\end{array}$ & $1.82 \pm 0.56^{*}$ & $1.03 \pm 0.21^{*}$ & $0.25 \pm 0.07^{*}$ & $1.12 \pm 0.21^{*}$ & $0.18 \pm 0.03^{*}$ & $0.26 \pm 0.07^{*}$ \\
\hline HFD+LD & $2.39 \pm 0.45$ & $1.16 \pm 0.13^{*}$ & $0.66 \pm 0.09^{*}$ & $1.02 \pm 0.19$ & $0.45 \pm 0.14^{*}$ & $0.26 \pm 0.05^{*}$ \\
\hline $\mathrm{HFD}+\mathrm{MD}$ & $1.83 \pm 0.29^{*}$ & $1.13 \pm 0.25^{*}$ & $0.44 \pm 0.14^{*}$ & $1.08 \pm 0.17^{*}$ & $0.38 \pm 0.11^{*}$ & $0.23 \pm 0.07^{*}$ \\
\hline $\mathrm{HFD}+\mathrm{HD}$ & $1.98 \pm 0.34^{*}$ & $0.87 \pm 0.15^{*}$ & $0.29 \pm 0.10^{*}$ & $1.04 \pm 0.11$ & $0.26 \pm 0.09^{*}$ & $0.21 \pm 0.02^{*}$ \\
\hline
\end{tabular}

Values are expressed as mean $\pm \mathrm{SD}(n=8)$; ${ }^{\#} p<0.05 v s$. the ND group and ${ }^{*} p<0.05 v s$. the HFD group. HAS: hydroxy- $\alpha$-sanshool; ND: normal diet; HFD: highfat diet; FNB: fenofibrate; LD: low dose of HAS; MD: medium dose of HAS; HD: high dose of HAS; LDL: low-density lipoprotein; HDL: low-density lipoprotein.

ND

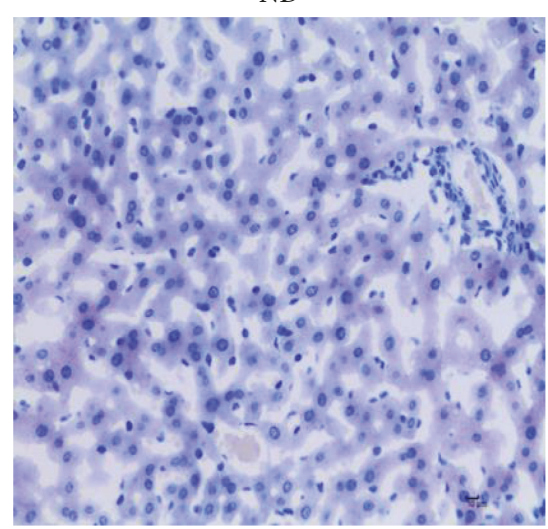

(a)

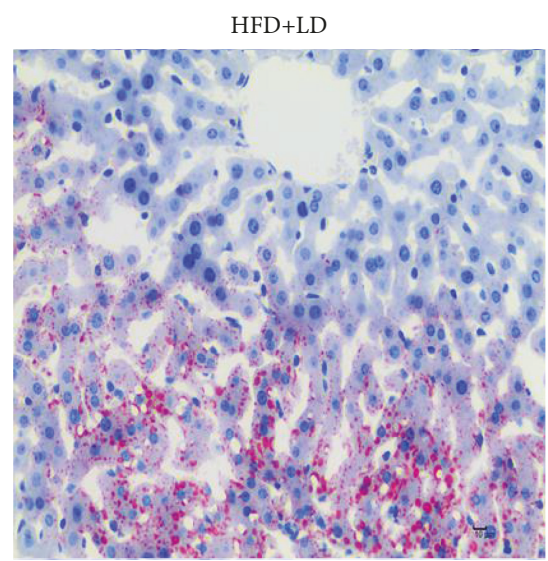

(d)

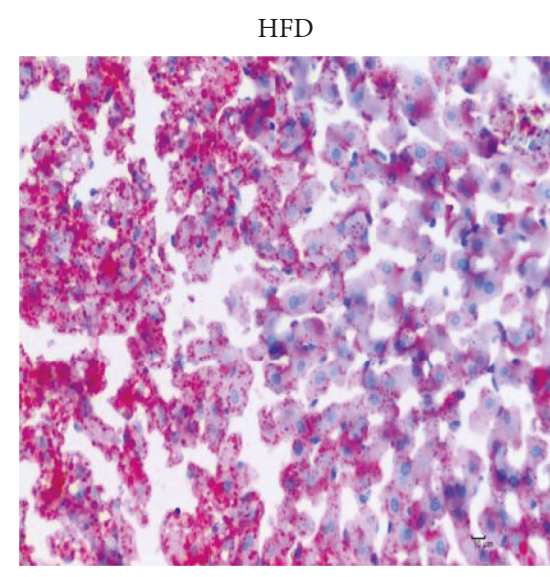

(b)

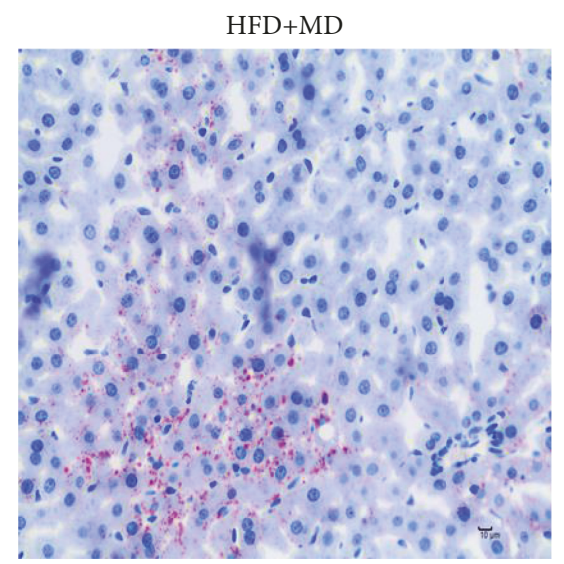

(e)

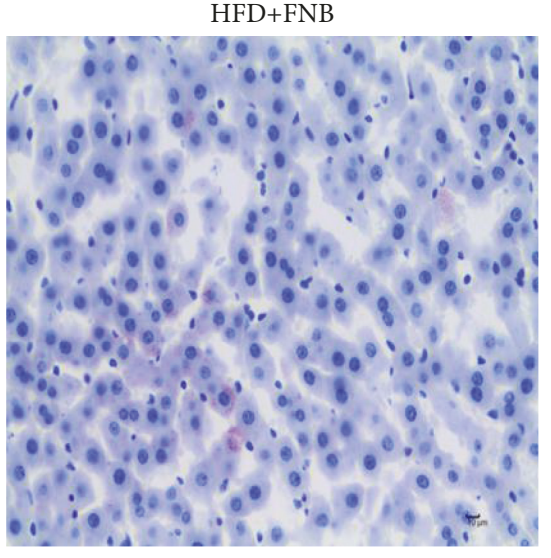

(c)

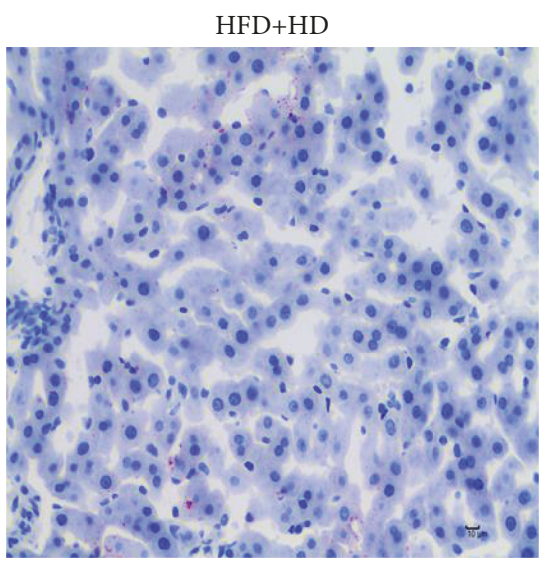

(f)

FIGURE 7: Histopathological examinations of liver tissues with oil red $\mathrm{O}$ staining. (a-f) represent oil red O-stained liver sections of rats in the ND, HFD, HFD+FNB, HFD+LD, HFD+MD, and HFD+HD groups, respectively. ND: normal diet-treated rats; HFD: high-fat diet-treated rats; HFD+FNB: HFD supplemented with fenofibrate-treated rats; HFD+LD: rats treated with HFD supplemented with a low dose of HAS; HFD+MD: rats treated with HFD supplemented with a middle dose of HAS; HFD+HD: rats treated with HFD supplemented with a high dose of HAS.

high-fat conditions (Figures 10(c) and 10(e)). The levels of all detected proteins were higher in the HFD+HAS groups (9, 18 , and $36 \mathrm{mg} / \mathrm{kg}$ ) compared with those in the HFD group (Figures $10(\mathrm{c})-10(\mathrm{e})$; all $p<0.05$ ), while the changes resulting from FNB treatment were nonsignificant.

\section{Discussion}

Traditional Chinese medicines (TCMs) have been used comprehensively to treat various diseases for thousands of years in Chinese folk medicine [13-15]. In addition, plants are 
ND

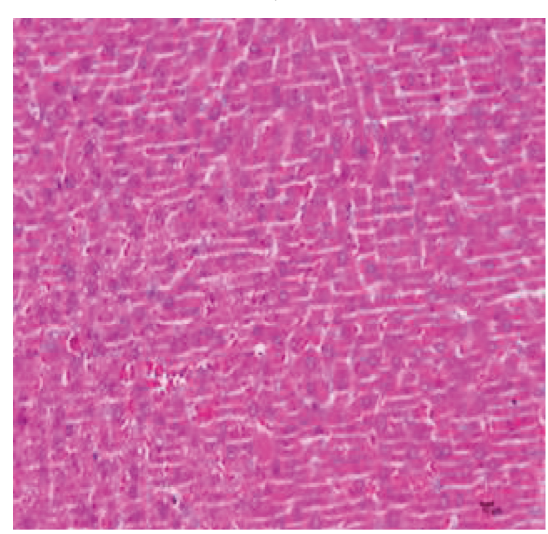

(a)

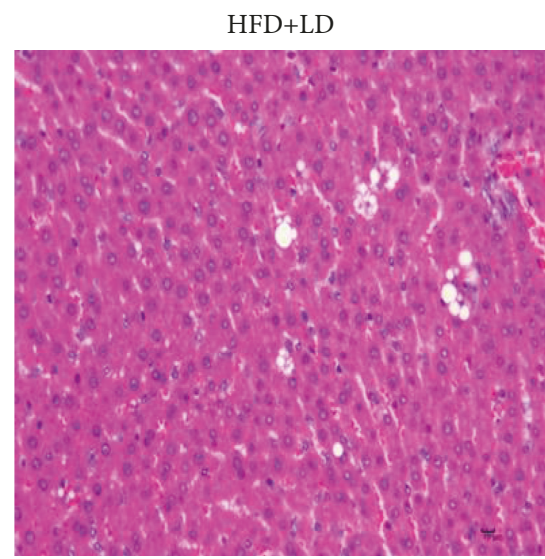

(d)
HFD

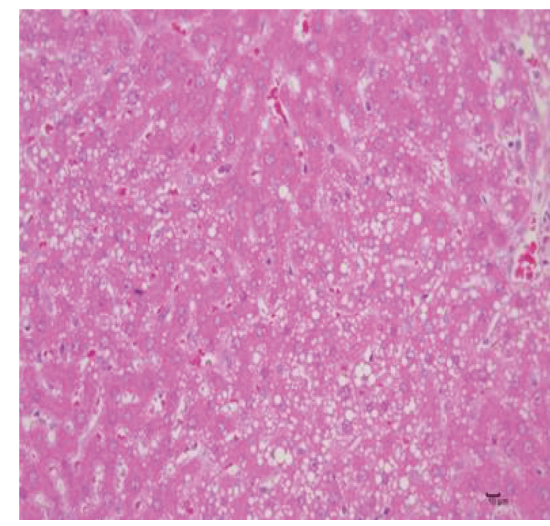

(b)

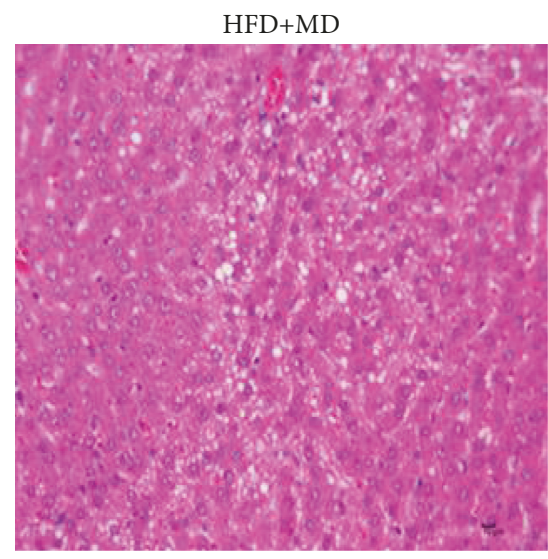

(e)

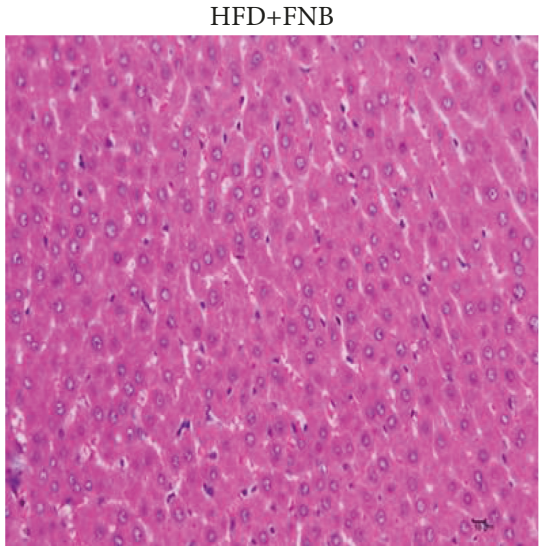

(c)

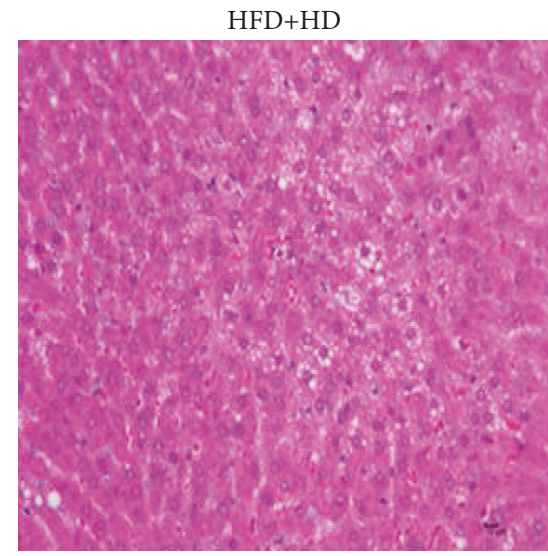

(f)

FIGURE 8: Histopathological examinations of liver tissues using H\&E staining. (a-f) represent H\&E-stained liver sections of rats in the ND, HFD, HFD+FNB, HFD+LD, HFD+MD, and HFD+HD groups, respectively. ND: normal diet-treated rats; HFD: high-fat diet-treated rats; HFD+FNB: HFD supplemented with fenofibrate-treated rats; HFD+LD: rats treated with HFD supplemented with a low dose of HAS; HFD+MD: rats treated with HFD supplemented with a middle dose of HAS; HFD+HD: rats treated with HFD supplemented with a high dose of HAS.

TABLE 4: Effect of HAS on MDA, GSH, and SOD in liver tissues.

\begin{tabular}{lccc}
\hline Groups & $\begin{array}{c}\text { SOD } \\
\text { (U/mgprot) }\end{array}$ & $\begin{array}{c}\text { MDA } \\
(\mu \mathrm{mol} / \text { gprot })\end{array}$ & $\begin{array}{c}\text { GSH } \\
(\mu \mathrm{mol} / \text { gprot })\end{array}$ \\
\hline ND & $204.74 \pm 15.52$ & $3.69 \pm 0.43$ & $35.274 \pm 2.26$ \\
HFD & $147.83 \pm 32.77^{\#}$ & $5.84 \pm 1.16^{\#}$ & $26.583 \pm 3.43^{\#}$ \\
HFD+FNB & $214.40 \pm 23.05^{*}$ & $3.68 \pm 0.79^{*}$ & $36.120 \pm 5.72^{*}$ \\
HFD+LD & $152.01 \pm 16.73^{*}$ & $3.88 \pm 093^{*}$ & $29.371 \pm 7.59$ \\
HFD+MD & $207.60 \pm 28.29^{*}$ & $3.82 \pm 081^{*}$ & $30.210 \pm 7.27$ \\
HFD+HD & $216.32 \pm 39.64^{*}$ & $3.37 \pm 061^{*}$ & $34.722 \pm 5.53^{*}$ \\
\hline
\end{tabular}

Values are expressed as mean \pm SD $(n=8) ;{ }^{\#} p<0.05 v s$. the ND group and ${ }^{*} p<0.05 v s$. the HFD group. HAS: hydroxy- $\alpha$-sanshool; ND: normal diet; HFD: high-fat diet; FNB: fenofibrate; LD: low dose of HAS; MD: medium dose of HAS; HD: high dose of HAS; SOD: superoxide dismutase; MDA: malondialdehyde; GSH: glutathione.

the predominant sources of food for humans and it is reported that over half of the available drugs are derived from natural products derived from plants $[16,17]$. Consequently, searching for candidate agents with reliable antiobesity and hypolipidemic activities from plants is a feasible approach. Importantly, in the present study, we reported that hydroxy- $\alpha$-sanshool (HAS) isolated from $Z$. bungeanum has potential antiobesity and hypolipidemic activities and attenuates liver oxidative stress effects.

Obesity is generally recognized as a chronic metabolic disorder and is characterized by excess body fat and weight induced by imbalance of energy consumption and intake [18]. Currently, obesity is believed to be closely related to high-fat diets, lack of exercise, and genetics $[13,19]$. In the present study, we prepared fatty and hyperlipidemic rats fed with high-fat diets, which had similar pathological characteristics to patients with obesity and hyperlipidemia. The results showed that compared with the model rats, treatment with HAS not only significantly decreased body weight gain but also reduced the underbelly visceral fat of the HFD-fed rats. In addition, fatty liver is a common complication of obesity and hyperlipidemia [20]. Based on the H\&E and oil red staining histopathological examinations, HAS administration could markedly ameliorate the degree of fatty liver of the HFD-fed rats. This evidence demonstrated that HAS possesses potential antiobesity and hypolipidemic activities in HFD-fed rats. 


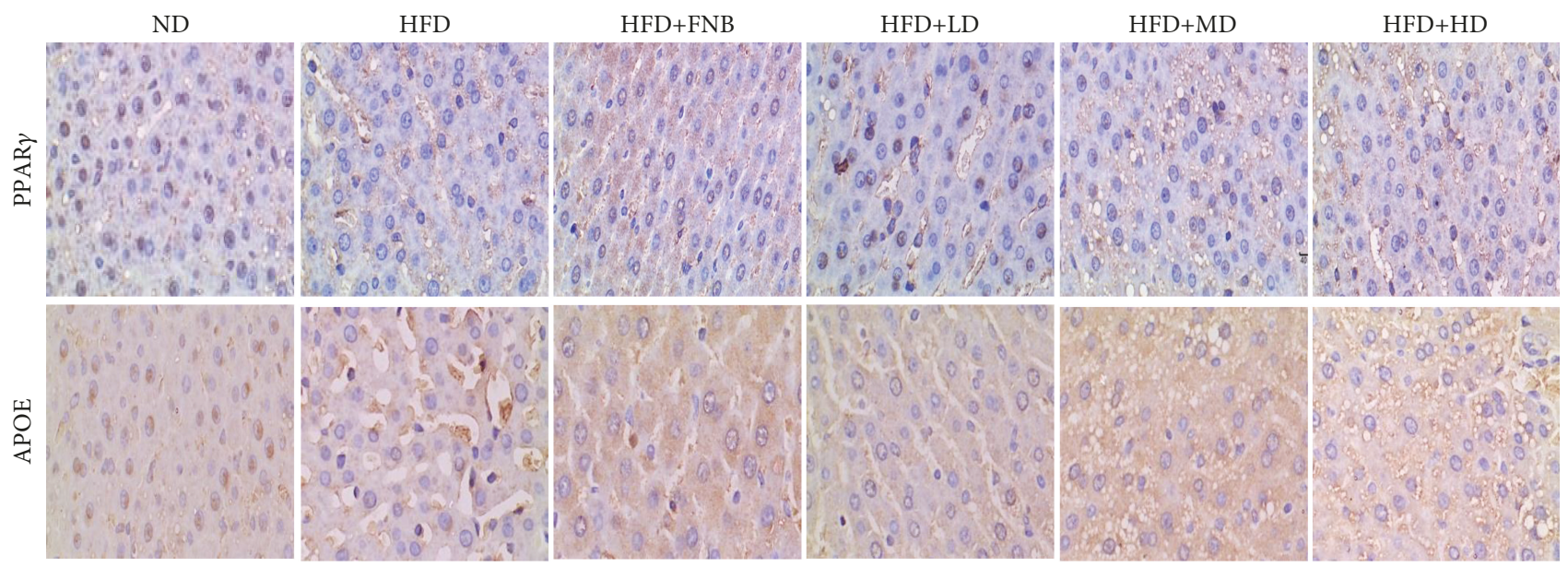

(a)

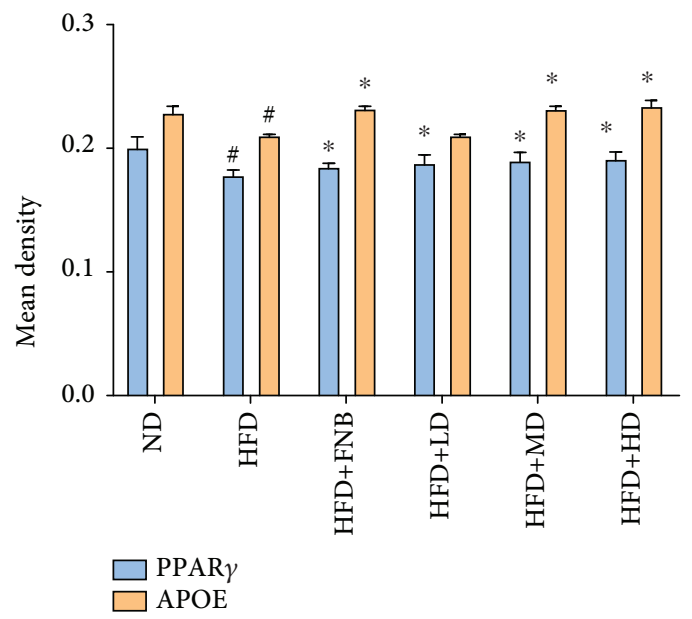

(b)

FIGURE 9: Effect of HAS on the expression of PPAR $\gamma$ and APOE in liver tissues using IHC. ND: normal diet-treated rats; HFD: high-fat diettreated rats; HFD+FNB: HFD supplemented with fenofibrate-treated rats; HFD+LD: rats treated with HFD supplemented with a low dose of HAS; HFD+MD: rats treated with HFD supplemented with a middle dose of HAS; HFD+HD: rats treated with HFD supplemented with a high dose of HAS; ${ }^{\#} p<0.05$ vs. ND; ${ }^{*} p<0.05$ vs. HFD; n.s.: nonsignificant.

Obesity induces lipid metabolism disorders, in particular the metabolism of TC, TG, LDL-C, and HDL-C. Clinically, patients with obesity and hyperlipidemia commonly present with increases in TC, TG, and LDL-C and decreased HDL-C $[21,22]$. Interestingly, the results of the present study demonstrated that HAS administration could decrease the contents of TC, TG, and LDL-C and increase the HDL-C contents in HFD rats.

Moreover, increasing evidence indicates that oxidative stress is closely correlated with the development of obesity, fatty liver, hyperlipidemia, and arteriosclerosis. In addition, inhibition of oxidative stress might be beneficial to control obesity and hyperlipidemia and protecting liver tissue [23, $24]$. MDA is one of the major end products of lipid peroxidation (LPO) and is commonly used as an indicator of liver tissue damage. In contrast, the antioxidant defense system in the body plays a vital role in inhibiting oxidative stress. GSH and SOD are crucial antioxidative enzymes [25] in liver tissues. Our study demonstrated that HAS administration could increase the levels of GSH and SOD and decrease the level of MDA in liver tissues. These results revealed that HAS could decrease the oxidative stress level in liver tissues.

Peroxisome proliferator-activated receptors (PPARs) play an important regulatory role in regulating the homeostasis of adipose tissue by regulating the balance between anabolic and oxidative processes [26]. PPARs are mainly expressed in white and brown adipose tissue, which plays an important anabolic role in promoting fat storage, fat formation, and thermogenesis [27, 28]. Furthermore, APOE, a protective protein for cardiovascular diseases, can interact with PPAR $\gamma$ to decrease cardiovascular disease risk [29]. Interestingly, the results of the mRNA analyses suggested that the HAS treatment increases the expression of Pparg and Apoe. The IHC and Western blotting results also indicated that HAS treatment could increase the levels of PPAR $\gamma$ and APOE in the nuclei, as a stress response of the body's defense system. These results indicated that HAS might regulate lipid metabolism and reduce oxidative stress in the 


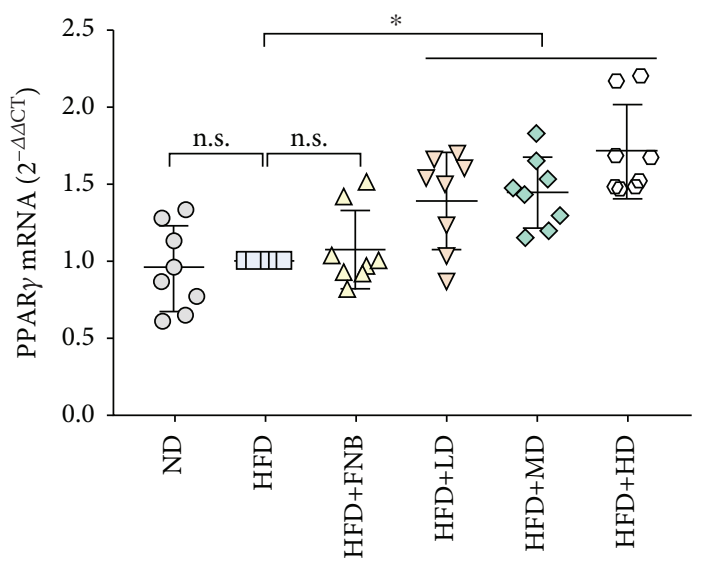

(a)

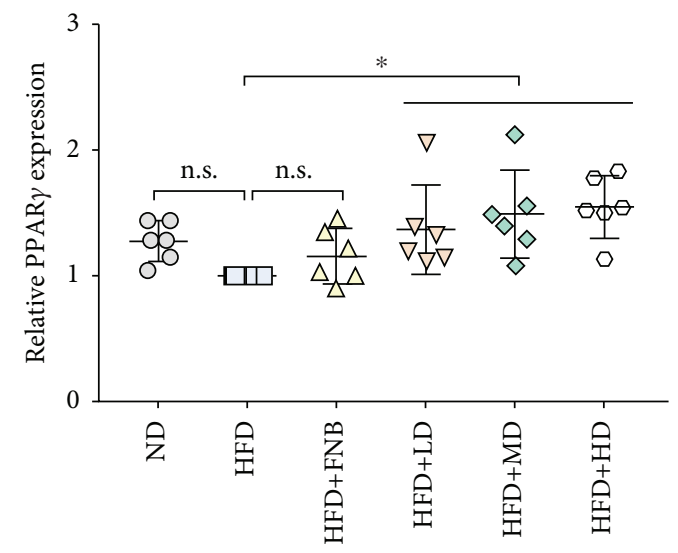

(c)

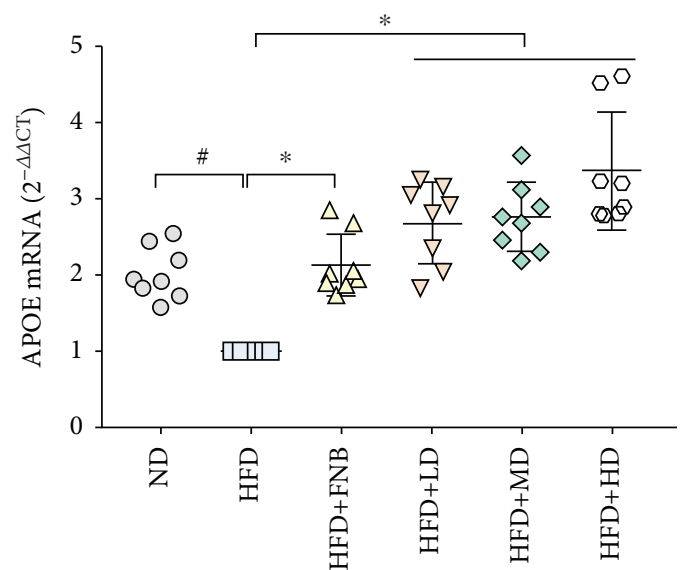

(b)

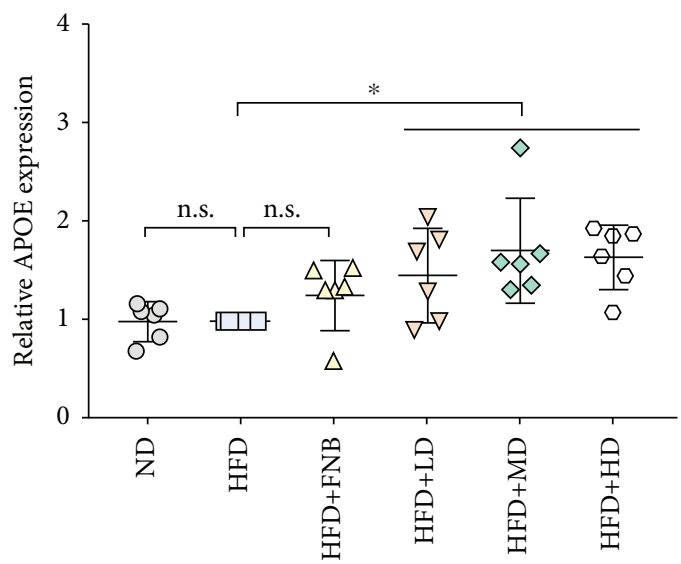

(d)

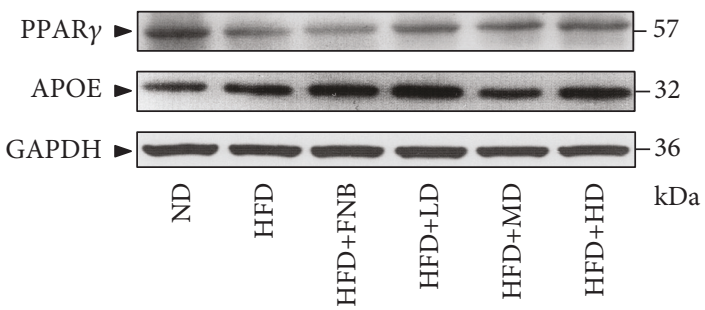

(e)

FIGURE 10: Effect of HAS on the transcription of genes involved in lipid metabolism in liver tissues (a, b); values are expressed as mean \pm SD $(n=9)$. Effect of HAS on the levels of PPAR $\gamma$ and APOE in liver tissue using Western blotting $(c-e)$. Values are expressed as mean \pm SD $(n=6)$. ND: normal diet-treated rats; HFD: high-fat diet-treated rats; HFD+FNB: HFD supplemented with fenofibrate-treated rats; HFD+LD: rats treated with HFD supplemented with a low dose of HAS; HFD+MD: rats treated with HFD supplemented with a middle dose of HAS; HFD+HD: rats treated with HFD supplemented with a high dose of HAS; ${ }^{*} p<0.05 v s$. ND; ${ }^{*} p<0.05 v s$. HFD; n.s.: nonsignificant.

high-fat state by upregulating Pparg expression. Meanwhile, HAS also increased the expression of Apoe and promoted the metabolism and transformation of lipoproteins.

\section{Conclusion}

In summary, hydroxy- $\alpha$-sanshool (HAS) could play a significant role in improving the lipid profile and may act as a protective agent against atherosclerosis by regulating of PPAR $\gamma$ and APOE to reduce lipid peroxidation. Therefore, HAS could be considered as a potential candidate drug to cure or prevent hyperlipidemia.

\section{Data Availability}

The data used to support the findings in this paper are available from the corresponding author upon request.

\section{Conflicts of Interest}

There are no conflicts of interest associated with this paper. 


\section{Acknowledgments}

This research was supported by the Project of Administration of Traditional Chinese Medicine of Sichuan Province of China (grant no. 2018JC001) and the Program of Science \& Technology Department of Sichuan Province (grant no. 2018SZ0064).

\section{References}

[1] D. S. Menees and E. R. Bates, "Evaluation of patients with suspected coronary artery disease," Coronary Artery Disease, vol. 21, no. 7, pp. 386-390, 2010.

[2] J. S. Lawton, "Sex and gender differences in coronary artery diseases," Seminars in Thoracic and Cardiovascular Surgery, vol. 23, no. 2, pp. 126-130, 2011.

[3] A. M. Durkar, R. R. Patil, and S. R. Naik, "Hypolipidemic and antioxidant activity of ethanolic extract of symplocos racemosa roxb. in hyperlipidemic rats: an evidence of participation of oxidative stress in hyperlipidemia," Indian Journal of Experimental Biology, vol. 52, no. 1, pp. 36-45, 2014.

[4] F. B. Hu, "Globalization of food patterns and cardiovascular disease risk," Circulation, vol. 118, no. 19, pp. 1913-1914, 2008.

[5] V. Matschke, C. Theiss, and J. Matschke, "Oxidative stress: the lowest common denominator of multiple diseases," Neural Regeneration Research, vol. 14, no. 2, pp. 238-241, 2019.

[6] R. D. Del, A. J. Stewart, and N. Pellegrini, "A review of recent studies on malondialdehyde as toxic molecule and biological marker of oxidative stress," Nutrition Metabolism \& Cardiovascular Diseases, vol. 15, no. 4, pp. 316-328, 2005.

[7] S. Y. Yang, L. Liu, L. Meng, and X. Y. Hu, "Capsaicin is beneficial to hyperlipidemia, oxidative stress, endothelial dysfunction, and atherosclerosis in Guinea pigs fed on a high-fat diet," Chemico-Biological Interactions, vol. 297, pp. 1-7, 2019.

[8] M. H. Pan, J. W. Chen, Z. L. Kong, J. C. Wu, C. T. Ho, and C. S. Lai, "Attenuation by tetrahydrocurcumin of adiposity and hepatic steatosis in mice with high-fat-diet-induced obesity," Journal of Agricultural and Food Chemistry, vol. 66, no. 48, pp. 12685-12695, 2019.

[9] L. Xiang, Y. Liu, C. Xie et al., "The chemical and genetic characteristics of szechuan pepper (Zanthoxylum bungeanum and Z. armatum) cultivars and their suitable habitat," Frontiers in Plant Science, vol. 7, 2016.

[10] M. M. Zhang, J. L. Wang, L. Zhu et al., "Zanthoxylum bungeanum Maxim. (Rutaceae): a systematic review of its traditional uses, botany, phytochemistry, pharmacology, pharmacokinetics, and toxicology," International Journal of Molecular Sciences, vol. 18, no. 10, p. 2172, 2018.

[11] Chinese Pharmacopoeia Commission, "Chinese pharmacopoeia," Science and Technology Press of Shanghai, vol. 1, pp. 159-160, 2015.

[12] T. Wu, L. Zhong, Z. Hong et al., "The effects of Zanthoxylum bungeanum extract on lipid metabolism induced by sterols," Journal of Pharmacological Sciences, vol. 127, no. 3, pp. 251259, 2014.

[13] H. Du, J. S. You, X. Zhao, J. Y. Park, S. H. Kim, and K. J. Chang, "Antiobesity and hypolipidemic effects of lotus leaf hot water extract with taurine supplementation in rats fed a high fat diet," Journal of Biomedical Science, vol. 17, Supplement 1, p. S42, 2010.
[14] Y. N. He, S. P. Ou, X. Xiong et al., "Stems and leaves of Aconitum carmichaelii Debx. as potential herbal resources for treating rheumatoid arthritis: chemical analysis, toxicity and activity evaluation," Chinese Journal of Natural Medicines, vol. 16, no. 9, pp. 644-652, 2018.

[15] B. Patwardhan, "Ethnopharmacology and drug discovery," Journal of Ethnopharmacology, vol. 100, no. 1-2, pp. 50-52, 2005.

[16] A. D. Kinghorn, Y. W. Chin, and S. M. Swanson, "Discovery of natural product anticancer agents from biodiverse organisms," Current Opinion in Drug Discovery \& Development, vol. 12, no. 2, pp. 189-196, 2010.

[17] W. Peng, H. Shen, B. Lin et al., "Docking study and antiosteoporosis effects of a dibenzylbutane lignan isolated from Litsea cubeba targeting Cathepsin K and MEK1," Medicinal Chemistry Research, vol. 27, no. 9, pp. 2062-2070, 2018.

[18] D. J. Newman and G. M. Cragg, "Natural products as sources of new drugs from 1981 to 2014," Journal of Natural Products, vol. 79, no. 3, pp. 629-661, 2016.

[19] W. J. Ren, Q. Q. Liu, T. Ren et al., "Effects of active ingredients of Zanthoxylum oleoresin on lipid metabolism in rats fed high fat diet," Acta Nutrimenta Sinica, vol. 3, no. 37, pp. 288-292, 2015.

[20] S. Spahis, F. Alvarez, N. Ahmed et al., "Non-alcoholic fatty liver disease severity and metabolic complications in obese children: impact of omega-3 fatty acids," The Journal of Nutritional Biochemistry, vol. 58, pp. 28-36, 2018.

[21] F. M. Perla, M. Prelati, M. Lavorato, D. Visicchio, and C. Anania, "The role of lipid and lipoprotein metabolism in non-alcoholic fatty liver disease," Children, vol. 4, no. 6, p. 46, 2017.

[22] J. Ren, S. M. Grundy, J. Liu et al., "Long-term coronary heart disease risk associated with very-low-density lipoprotein cholesterol in Chinese: the results of a 15-year Chinese multiprovincial cohort study (CMCS)," Atherosclerosis, vol. 211, no. 1, pp. 327-332, 2010.

[23] Q. R. Jiang, C. L. Yao, and G. Z. Li, "Efects of lyeium barbarum polysaeeharides on blood lipid and oxidative stress of aorta in hyperlipidemia rats," Ningxia Medicine Journal, vol. 32, no. 3, pp. 504-506, 2010.

[24] B. B. Ma, C. Bian, B. Hong et al., "Research progress in small molecule drugs based on high-density lipoprotein for anti-atherosclerosis," Acta Pharmaceutica Sinica, vol. 53, no. 3, pp. 328-335, 2018.

[25] W. Peng, X. Q. Qiu, Z. H. Shu et al., "Hepatoprotective activity of total iridoid glycosides isolated from Paederia scandens (Lour.) Merr. var. Tomentosa," Journal of Ethnopharmacology, vol. 174, pp. 317-321, 2015.

[26] P. Corrales, A. Vidalpuig, and G. Medinagómez, "Ppars and metabolic disorders associated with challenged adipose tissue plasticity," International Journal of Molecular Sciences, vol. 19, no. 7, article 2124, 2018.

[27] R. Siersbaek, R. Nielsen, and S. Mandrup, "PPAR $\gamma$ in adipocyte differentiation and metabolism-novel insights from genome-wide studiest," FEBS Letters, vol. 584, no. 15, pp. 3242-3249, 2010.

[28] P. Ferre, "The biology of peroxisome proliferator-activated receptors: relationship with lipid metabolism and insulin sensitivity," Diabetes, vol. 53, Supplement 1, pp. S43-S50, 2004.

[29] D. Q. Peng, S. P. Zhao, S. Nie, and J. Li, "Gene-gene interaction of PPAR $\gamma$ and apoe affects coronary heart disease risk," International Journal of Cardiology, vol. 92, no. 2-3, pp. 257263,2006 


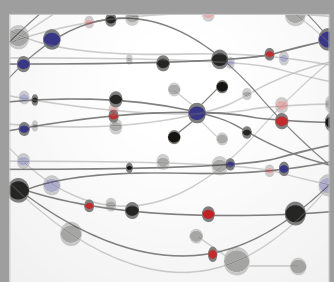

The Scientific World Journal
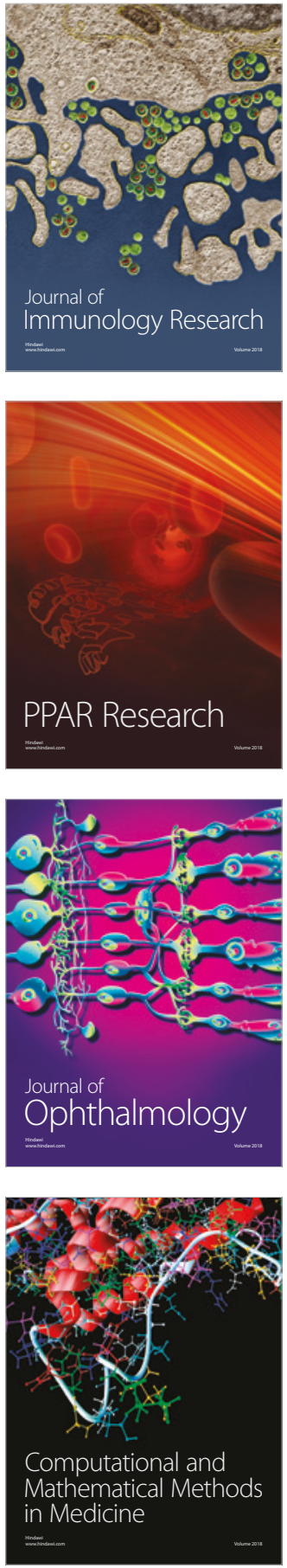

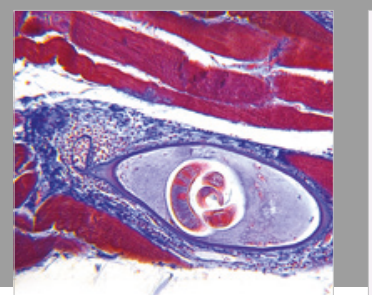

Gastroenterology Research and Practice

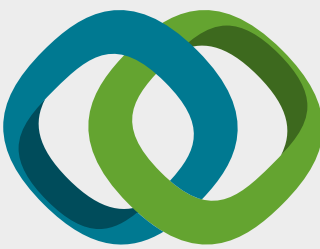

\section{Hindawi}

Submit your manuscripts at

www.hindawi.com
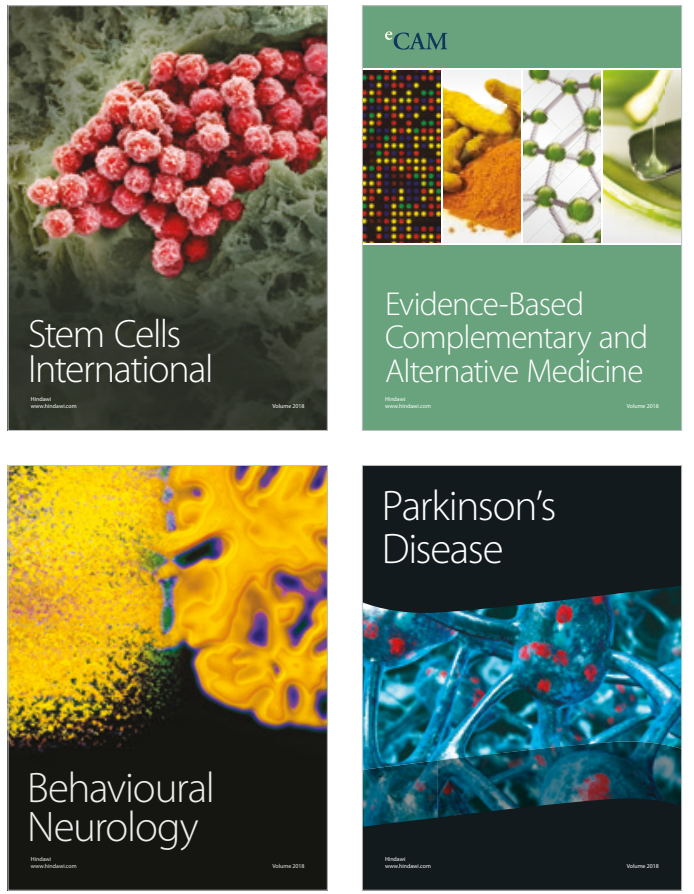

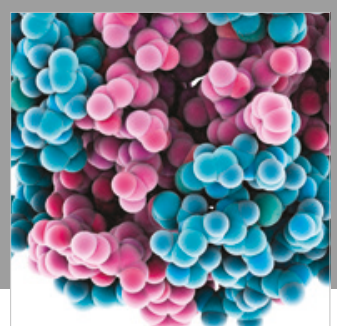

ournal of

Diabetes Research

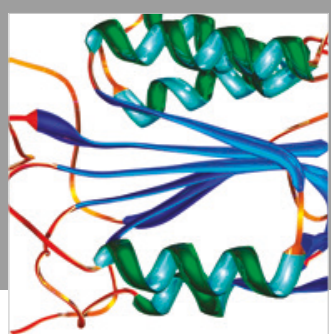

Disease Markers
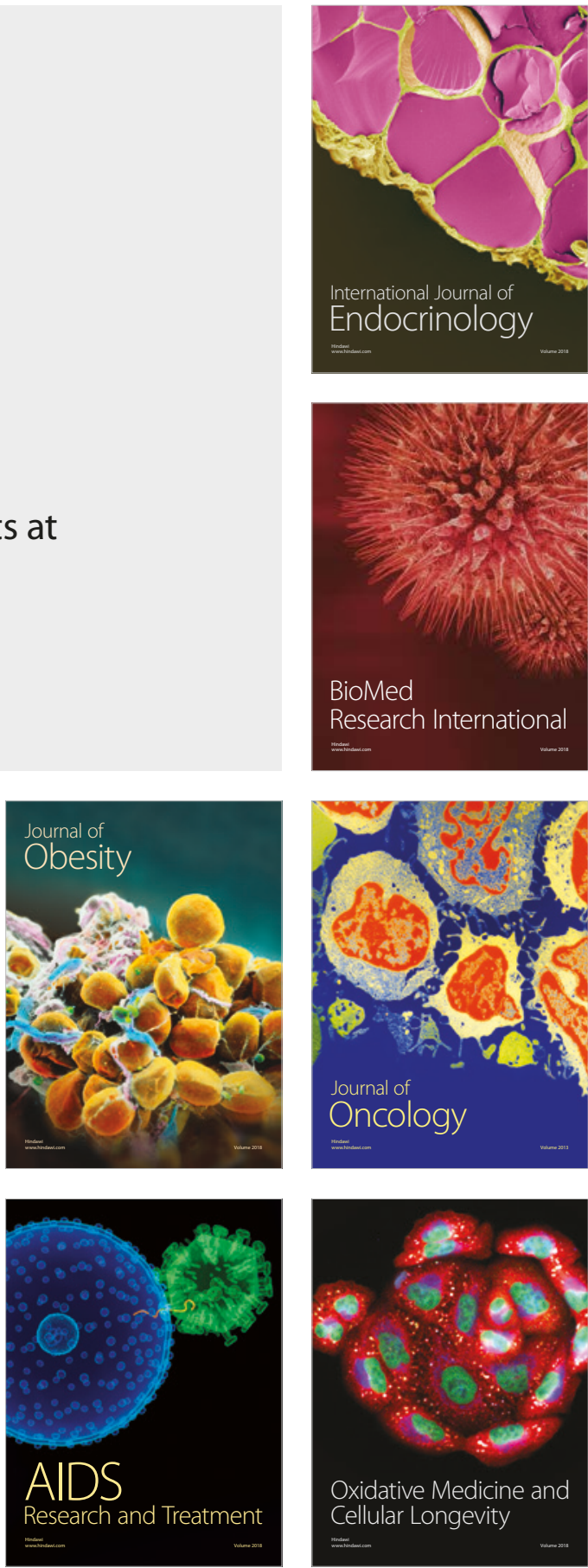